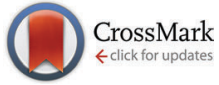

Cite this: Phys. Chem. Chem. Phys., 2015, 17, 19656

Received 5th May 2015, Accepted 30th June 2015

DOI: $10.1039 / \mathrm{c} 5 \mathrm{cp} 02615 f$

www.rsc.org/pccp

\section{Vibrationally resolved optical spectra of modified diamondoids obtained from time-dependent correlation function methods}

\author{
Shiladitya Banerjee, Tony Stüker and Peter Saalfrank*
}

Optical properties of modified diamondoids have been studied theoretically using vibrationally resolved electronic absorption, emission and resonance Raman spectra. A time-dependent correlation function approach has been used for electronic two-state models, comprising a ground state $(\mathrm{g})$ and a bright, excited state (e), the latter determined from linear-response, time-dependent density functional theory (TD-DFT). The harmonic and Condon approximations were adopted. In most cases origin shifts, frequency alteration and Duschinsky rotation in excited states were considered. For other cases where no excited state geometry optimization and normal mode analysis were possible or desired, a short-time approximation was used. The optical properties and spectra have been computed for (i) a set of recently synthesized $\mathrm{sp}^{2} / \mathrm{sp}^{3}$ hybrid species with $\mathrm{C}=\mathrm{C}$ double-bond connected saturated diamondoid subunits, (ii) functionalized (mostly by thiol or thione groups) diamondoids and (iii) urotropine and other $\mathrm{C}$-substituted diamondoids. The ultimate goal is to tailor optical and electronic features of diamondoids by electronic blending, functionalization and substitution, based on a molecular-level understanding of the ongoing photophysics.

\section{Introduction}

Diamondoids form a family of hydrocarbons, consisting of repeated units of connected cyclohexane rings. ${ }^{1-3}$ The first member of this series is adamantane, $\mathrm{C}_{10} \mathrm{H}_{16}$, a saturated diamondoid with $\mathrm{sp}^{3}$-hybridized carbon atoms. Higher homologues are known as diamantane, $\mathrm{C}_{14} \mathrm{H}_{20}$, triamantane $\mathrm{C}_{18} \mathrm{H}_{24}$, tetramantane $\mathrm{C}_{22} \mathrm{H}_{28}$, pentamantane and so on. Diamondoids are chemically quite inert, hard compounds. ${ }^{4}$ They are good (monochromatic) electron emitters with negative electron affinity, ${ }^{5-7}$ transparent wide-gap materials, absorbing light significantly around and above about $6 \mathrm{eV},{ }^{8-10}$ i.e., in the UV. Diamondoids are excellent fluorescing materials, known to fluoresce both in the solid state and in the gas phase. For absorption to and fluorescence from, the lowest electronic excited states, often pronounced vibrational finestructures in spectra have been observed. ${ }^{10}$ Vibrations involving excited states are also important for the interpretation of resonance Raman spectra, another important tool to characterize diamondoids. ${ }^{11}$ In general, vibronic spectra are powerful tools to unravel details of optical properties of diamondoids and the focus of this paper.

Due to the diverseness of their shape and composition and the ability to be functionalized (see below), one hopes that diamondoids have tunable optical and electronic properties for

Institut für Chemie, Universität Potsdam, Karl-Liebknecht-Straße 24-25, D-14476 Potsdam-Golm, Germany.E-mail: peter.saalfrank@uni-potsdam.de possible applications. Ref. 12 reported the effects of $\mathrm{C}-\mathrm{H}$ and interstitial substitution on the HOMO-LUMO energy and the band gaps of a range of diamondoids, using density functional theory computations. Along these lines, the synthesis of artificial diamondoids has greatly advanced in recent years, ${ }^{13}$ as well as their spectroscopic investigation. ${ }^{14-18}$ The modified diamondoids studied in these works can roughly be classified according to three main categories:

(i) "Electronically blended diamondoids", i.e., diamondoid subunits connected to each other by $\mathrm{sp}^{2}$-hybridized $\mathrm{C}$ atoms (or other unsaturated units). The subunits may be comprised of the same (Dia = Dia, two diamantanes; Ada $=$ Ada, two adamantanes) or different (Ada $=$ Dia, Ada $=$ Dia $=$ Ada) diamondoid molecules. Several of these (and related) molecules have been synthesized by Schreiner and co-workers. ${ }^{11,13}$ For example, Dia $=$ Dia which exists as $E$ and $Z$ stereoisomers has been the subject of recent physico-chemical ${ }^{11}$ and theoretical ${ }^{18}$ characterization. In Dia = Dia, a greatly reduced HOMO-LUMO gap is found compared to pristine diamondoids, as well as a strongly enhanced $\mathrm{C}=\mathrm{C}$ vibration in resonance Raman. Among other experimental studies, the valence photoelectron spectra of selected diamondoids, joined by single or double $\mathrm{C}=\mathrm{C}$ bonds have been measured in the recent past. ${ }^{19}$

To extend this work and identify trends in $\mathrm{C}=\mathrm{C}$-blended diamondoids, other and also more complicated species such as $\mathrm{Ada}=\mathrm{Dia}=\mathrm{Ada}$ (with two $\mathrm{C}=\mathrm{C}$ double bonds) will be considered here. 
(ii) "Functionalized diamondoids", ${ }^{15-17}$ i.e., species where one or multiple $\mathrm{H}$ atom(s) were substituted by particular functional groups like hydroxyl, cyano, amino, or thiol $(-\mathrm{SH})$ groups. Sulfur-containing diamondoids are particularly interesting because of the ease of their attachment to metal surfaces; their synthesis dates back nearly to a decade. ${ }^{20,21}$ It is possible to attach multiple functional groups, resulting in, e.g., dithiol, trithiol, and so on. Structural isomers also exist, depending on the position of the functional group (e.g., adamantane-1-thiol and adamantane-2-thiol) or the relative positions of two or more functional groups (e.g., adamantane-1,2-dithiol and adamantane1,3-dithiol). Recently, Landt and co-workers showed that the incorporation of a thiol group in adamantane lowers its optical gap. ${ }^{15}$ This opens up the speculation on tunable optical properties of diamondoids by thiolization.

To identify trends in mono- $v s$. di-substitution as well as effects of the position of the functional group(s), on the optical gaps and the vibronic absorption, emission and resonance Raman spectra, various thiol- and dithiol-adamantanes will be studied in this work.

Also, the substitution (of two $\mathrm{H}$ atoms) by $=\mathrm{S}$ groups, leading to thiones, e.g. adamantane thione, $\mathrm{C}_{10} \mathrm{H}_{14} \mathrm{~S}$, have been discussed in the literature as possible routes towards "tuned" materials. In a recent theoretical work, ${ }^{22}$ Vörös and co-workers predicted by PBE0/cc-pVTZ calculations for adamantane and $[1(2,3) 4]$-pentamantane, that the optical gap is reduced gradually by the systematic substitution of thione groups. For adamantane1,2,5,6-tetrathione and adamantane hexathione, for example, the optical gap was estimated to be in the visible range, between 2-3 eV.

No vibronic effects and real spectra were considered in that work, however, which we will do here for selected thionized diamondoids.

As a sideline, also several adamantanes functionalized with alcohol $(-\mathrm{OH})$ and bromo $(-\mathrm{Br})$ groups will be studied.

(iii) "Doping” or "C-substituting" diamondoids", i.e., replacing one or more methine $(\mathrm{CH})$ or methylene $\left(\mathrm{CH}_{2}\right)$ groups of pristine diamondoids by iso-electronic groups such as $\mathrm{N}$ and $\mathrm{O}$, is the final route of tuning properties of diamondoids to be studied here. This can lead to the formation of molecules with entirely new electronic and optical properties. For instance, substitution of the four $-\mathrm{CH}$ groups of adamantane by the iso-electronic $\mathrm{N}$ atom results in urotropine or hexamethylene tetramine, $\left(\mathrm{CH}_{2}\right)_{6} \mathrm{~N}_{4}$. In ref. 14, experiment has shown that the absorption and fluorescence of urotropine are very distinct from those of adamantane, with a "smooth", redshifted absorption band with no pronounced vibrational finestructure.

In the present work, we shall study some of the photophysical properties of urotropine by electronic structure methods, as well as of several other $\mathrm{N}$ - or O-substituted adamantanes.

Our work focuses on comparing vibrationally resolved absorption, emission and resonance Raman (rR) spectra of representative diamondoids from each of the three categories mentioned, using a two-state model with the ground state $\left(\mathrm{g}\right.$, or $\left.\mathrm{S}_{0}\right)$ and one bright, excited state (e, usually $\mathrm{S}_{1}$ ). In certain cases also a wider range of excited states was considered, then, however, mostly only for vertical electronic excitation energies. The ground and excited states are calculated by hybrid density functional theory (DFT) and linear-response time-dependent DFT (TD-DFT), respectively. Pristine diamondoids will be used as a reference. We shall also compare to experiment where possible. Our ultimate goal is to understand the photophysics of modified diamondoids on a fundamental level, and - based on this understanding - to help developing criteria for the "tuning", of optoelectronic properties of these versatile materials.

In order to arrive at vibrationally resolved spectra, we use a time-dependent correlation function approach as pioneered in chemical physics by Heller and co-workers. ${ }^{23,24}$ The timedependent approach can offer computational advantages by avoiding the computation of Franck-Condon factors. In particular in the harmonic approximation which we use here, quasianalytic expressions are available for auto- and cross-correlation functions. By combining (TD-)DFT with the correlation function approach in harmonic approximation, vibronically resolved spectra become available for medium-sized molecules with moderate computational effort and acceptable accuracy. The largest molecule treated here is $\mathrm{Ada}=\mathrm{Dia}=\mathrm{Ada}, \mathrm{C}_{34} \mathrm{H}_{44}$, with 228 normal modes. Other diamondoids have been studied elsewhere with the same methodology ${ }^{18}$ and bigger molecules of different type, e.g., $\beta$-carotene $\left(\mathrm{C}_{40} \mathrm{H}_{56}\right)$, in ref. 25 . In that reference, also the inclusion of Duschinsky rotation for resonance Raman spectra when calculated with time-dependent correlation functions has been put forward. (The further extension of the time-dependent approach to Herzberg-Teller corrections for resonance Raman was realized in ref. 26 and 27.) Duschinksy rotation, i.e., the rotation of normal modes in the electronically excited state relative to ground state modes, is an effect which will be considered here in many but not all examples.

The paper is organized as follows. The methods, models and approximations used for the computation of the spectra are described in Section II. Results are presented and discussed in Section III, for electronically blended diamondoids (Section III A), thiol- and thione-substituted diamondoids (Section III B) and urotropine (Section III C). Section IV summarizes the work and provides an outlook for possible future investigations in this field.

\section{Methods}

The vibrationally resolved absorption, emission and resonance Raman spectra have been calculated using the time-dependent correlation function approach, as popularized by Heller and co-workers. ${ }^{23,24}$ Here we work in harmonic and Condon approximations, using two-state models. Normal-mode coordinates are used throughout (rather than curvilinear coordinates, which can lead to somewhat different results ${ }^{28}$ ), the temperature is $0 \mathrm{~K}$ and possible effects of an environment are neglected.

In a first, more sophisticated model, called here the IMDHOFAD (independent mode displaced harmonic oscillator with frequency alteration and Duschinsky rotation) method, full geometry optimizations are carried out for the ground (g) and the selected bright, electronically excited state (e) and normal mode 
analyses are performed for both. Frequency alterations in the excited state and the Duschinsky rotation are taken fully into account. The method is described in more detail in ref. 18 and 25 and therefore only briefly reiterated below. In situations where optimization of excited states is not so trivial, unphysical, or simply unwanted (in order to save computational effort and allow for screening many molecules), the IMDHO (STA) approach will be used instead. In this approach, ${ }^{29}$ frequency alteration and Duschinsky rotation are not accounted for, and in addition the "short-time approximation" (STA) is used. Excited-state displacements are obtained from a local extrapolation scheme.

The first approach of above which is based on two fully optimized harmonic potentials, belongs to a broader class of models called "adiabatic". The latter approach, on the other hand, belongs to so-called "vertical" methods for which only information (on gradients and/or Hessians) at a Franck-Condon point for vertical transitions between two potentials enters. The vertical approach is not only more economic as it avoids excitedstate optimizations, it can also be of advantage if the excited state optimization is difficult, for practical or principal reasons, and then it is sometimes physically also more sensible. This is particularly so when large-amplitude motions and/or geometry displacements in excited states take place. ${ }^{28}$

For the adiabatic, IMDHOFAD model optimizations and normal mode analyses have been performed using density functional theory (DFT) and linear-response, time-dependent DFT (TD-DFT). Specifically, the B3LYP hybrid functional ${ }^{30,31}$ together with a triple zeta valence polarized (TZVP, ${ }^{32}$ ) basis set has been used throughout, if not explicitly stated otherwise. The validity of this method for diamondoids (good ratio of accuracy and computational effort), has been proven in ref. 18 . The GAUSSIAN09 ${ }^{33}$ quantum chemistry package has been used for optimizations and normal mode analyses. A FORTRAN code developed earlier ${ }^{25}$ was used to calculate the Duschinsky matrix and the dimensionless origin shifts between the normal modes of the two electronic states. The code is then used to calculate the auto-correlation functions and cross-correlation functions using the time-dependent approach. Spectra are obtained from Fourier transformed correlation functions by using the FFTW (Fastest Fourier Transform in the West) package. ${ }^{34}$ The IMDHO (STA) calculations, on the other hand, have been carried out directly with the quantum chemical package ORCA, ${ }^{35}$ on the (TD-)B3LYP/TZVP level of theory as well.

In the time-domain, the absorption cross-section is expressed as a Fourier transform of an autocorrelation function (we use atomic units in what follows $)^{18,23}$

$$
\begin{aligned}
\sigma_{\text {abs }}\left(\omega_{\mathrm{L}}\right)= & \frac{4 \pi \omega_{\mathrm{L}}}{3 c}\left|\underline{\mu}_{\text {eg }, 0_{\mathrm{g}}}\right|^{2} \operatorname{Re} \int_{0}^{\infty}\left\langle\phi_{0}^{\mathrm{g}}(0) \mid \mathrm{e}^{-i \hat{H}_{\mathrm{e}} t} \phi_{0}^{\mathrm{g}}(0)\right\rangle \\
& \exp \left[i\left(\omega_{\mathrm{L}}-E_{0}+E_{0}^{\mathrm{g}}\right) t\right] \exp (-\Gamma t) \mathrm{d} t
\end{aligned}
$$

where $\omega_{\mathrm{L}}$ is the frequency of the excitation radiation, $c$ is the velocity of light in vacuum and $E_{0}$ is the adiabatic minima separation energy between the ground and excited states. $E_{0}^{g}$ is the zero point energy of the initial vibrational state of the ground electronic state, and $\underline{\mu}_{\mathrm{eg}, 0_{\mathrm{g}}}$ the transition dipole moment between $\mathrm{g}$ and e states, which is taken constant and equal to the value at the equilibrium geometry $\left(0_{\mathrm{g}}\right)$ of the ground state, in the Condon approximation. Further, $\left|\phi_{0}^{\mathrm{g}}(0)\right\rangle$ is a product vibrational, initial wavefunction comprising $3 N-6$ vibrational ground state normal modes of the electronic ground state ( $N$ is the number of atoms). $\Gamma$ is the width (of the Lorentzian) used for damping the autocorrelation function, $\left\langle\phi_{0}^{\mathrm{g}}(0) \mid \mathrm{e}^{-i \hat{H}_{\mathrm{e}} t} \phi_{0}^{\mathrm{g}}(0)\right\rangle$. $\hat{H}_{\mathrm{e}}$ is the field-free, excited state nuclear Hamiltonian. In the harmonic approximation (even in the IMDHOFAD model), the expressions for multi-dimensional autocorrelation functions can be obtained analytically. ${ }^{23,25}$ Their computation requires optimized geometries of ground and excited states, ground and excited state normal frequencies $\omega_{1}^{\mathrm{g}}, \omega_{2}^{\mathrm{g}}, \ldots, \omega_{3 N-6}^{\mathrm{g}} ; \omega_{1}^{\mathrm{e}}, \omega_{2}^{\mathrm{e}}, \ldots, \omega_{3 N-6}^{\mathrm{e}}$ and corresponding normal modes and, derived from these quantities, the Duschinsky matrix and the so-called dimensionless origin shifts for modes.

The emission cross-section can also be expressed as the Fourier transform of a time-dependent correlation function

$$
\begin{aligned}
\sigma_{\mathrm{emi}}\left(\omega_{\mathrm{E}}\right)= & \frac{4 \pi \omega_{\mathrm{E}}^{3}}{3 c^{3}}\left|\underline{\mu}_{\mathrm{eg}, 0_{\mathrm{e}}}\right|^{2} \operatorname{Re} \int_{0}^{\infty}\left\langle\phi_{0}^{\mathrm{e}}(0) \mid \mathrm{e}^{-i \hat{H}_{\mathrm{g}} t} \phi_{0}^{\mathrm{e}}(0)\right\rangle \\
& \exp \left[i\left(\omega_{\mathrm{E}}+E_{0}+E_{0}^{\mathrm{e}}\right) t\right] \exp (-\Gamma t) \mathrm{d} t
\end{aligned}
$$

where $\omega_{\mathrm{E}}$ is the frequency of the emitted radiation, $E_{0}^{\mathrm{e}}$ the zero point energy of the excited electronic state, $\left|\phi_{0}^{\mathrm{e}}(0)\right\rangle$ the corresponding product vibrational state, from which the emission takes place. $\left|\mathrm{e}^{-i \hat{H}_{g} t} \phi_{0}^{\mathrm{e}}(0)\right\rangle$ is the time-dependent wave-packet obtained by propagation of $\left|\phi_{0}^{\mathrm{e}}\right\rangle$ under the influence of the field-free, ground state nuclear Hamiltonian $\hat{H}_{\mathrm{g}}$. Further, $\underline{\mu}_{\mathrm{eg}, 0_{\mathrm{e}}}$ is the transition dipole moment, evaluated now at the optimized geometry of the excited state $\left(0_{\mathrm{e}}\right)$. Like absorption, expressions for multi-dimensional autocorrelation functions can be obtained quasi-analytically. $^{23,25}$

The resonance Raman (rR) cross-section is calculated using

$$
\sigma_{\mathrm{rR}}^{i \rightarrow f}\left(\omega_{\mathrm{L}}, \omega_{\mathrm{S}}\right)=\frac{8 \pi \omega_{\mathrm{L}} \omega_{\mathrm{s}}{ }^{3}}{9 c^{4}} \sum_{q, q^{\prime}}\left|\alpha_{i \rightarrow f}^{q q^{\prime}}\left(\omega_{\mathrm{L}}\right)\right|^{2}
$$

where $\omega_{\mathrm{L}}$ is the frequency of the exciting light, $\omega_{\mathrm{S}}$ the frequency of the scattered radiation and $\alpha_{i \rightarrow f}^{q q^{\prime}}$ is the Raman polarizability for $\mathrm{rR}$ scattering from vibrational level $i$ to $f$, in the ground electronic state. For $0 \rightarrow 1$ Raman scattering as considered in this work, $i=0, f=1$. $q$ and $q^{\prime}$ refer to the $x, y$ or $z$ directions, along which the components of the transition dipole moment are considered. In the time-dependent regime, the Raman polarizability can be calculated as a Fourier transform of a crosscorrelation function ${ }^{18,24}$

$$
\begin{aligned}
\alpha_{i \rightarrow f}^{q q^{\prime}}\left(\omega_{\mathrm{L}}\right)= & \mu_{\mathrm{eg}, 0_{\mathrm{g}}}^{q} \mu_{\mathrm{eg}, 0_{\mathrm{g}}}^{q^{\prime}} \int_{0}^{\infty}\left\langle\phi_{f}^{\mathrm{g}}(0) \mid \mathrm{e}^{-i \hat{H}_{\mathrm{e}} t} \phi_{i}^{\mathrm{g}}(0)\right\rangle \\
& \exp \left[i\left(\omega_{\mathrm{L}}-E_{0}+E_{i}^{\mathrm{g}}\right) t\right] \exp (-\tilde{\Gamma} t) \mathrm{d} t .
\end{aligned}
$$

The quantity $\left\langle\phi_{f}^{\mathrm{g}}(0) \mid \mathrm{e}^{-i \hat{H}_{\mathrm{e}} t} \phi_{i}^{\mathrm{g}}(0)\right\rangle$ is the cross-correlation function. Similar to the autocorrelation functions, analytical expressions also exist for the evaluation of cross-correlation functions in multi-dimensions. ${ }^{24,25}$ Note that a different broadening factor $\tilde{\Gamma}$ 
is usually adopted for rR spectra. Here we compute rR spectra by fixing the excitation wavelengths $\omega_{\mathrm{L}}$ at certain values close to electronic excitation energies, as a function of the Raman shift. For $0 \rightarrow 1$ Raman scattering, we then obtain a stick spectrum as a function of the ground state vibrational frequencies, which is broadened here by Lorentzians of appropriate FWHM.

As outlined above, the time-dependent approach offers a variant, the short-time approximation (STA) which can be applied for absorption, emission and rR intensities. ${ }^{24,29}$ As stated, the STA is not always an approximation but may, as a representative of the "vertical" models, in certain special situations be even more accurate than the full IMDHOFAD model. In the STA approach to absorption as implemented in ORCA, ${ }^{29}$ excited-state energies are calculated for a particular set of geometries at and around the ground state geometry and quadratic fits to these energies are used to estimate a harmonic excited surface without explicitly optimizing its geometry and performing a normal mode analysis. Rather, this method neglects frequency alterations and modemixing in the excited state and will therefore be referred to as the IMDHO (STA) approach in what follows. Specifically, mode displacements are calculated using the relation, for absorption ${ }^{29}$

$$
\delta_{m}=-\left.\left(1 / \omega_{m}^{\mathrm{e}}\right)^{2} \frac{\partial V_{\mathrm{e}}^{\mathrm{e}}}{\partial Q_{m}^{\mathrm{g}}}\right|_{0_{\mathrm{g}}} .
$$

Here, $\left.\frac{\partial V_{\mathrm{e}}}{\partial Q_{m}^{0}}\right|_{\mathrm{g}_{\mathrm{g}}}$ is the gradient of the excited state potential energy $V_{\mathrm{e}}$ along the $m$ th mass weighted normal coordinate of a ground state mode, at the equilibrium geometry of the ground state (Franck-Condon point) and $\omega_{m}^{\mathrm{e}}$ is the excited state frequency of the $m$ th normal mode. In the IMDHO model, excited state frequencies are assumed to be the same as the ground state frequencies $\omega_{m}^{\mathrm{g}}$. The dimensionless shift of the $m$ th normal mode, $\Delta_{m}$ is then obtained from the relation

$$
\Delta_{m}=\delta_{m} \sqrt{\omega_{m}^{\mathrm{e}} / \hbar}
$$

The values obtained are used to calculate the absorption spectra, and similar procedures exist for emission and resonance Raman spectra. $^{29}$

\section{Results}

\section{A. Electronically blended diamondoids}

Let us first study molecules containing one or two $\mathrm{C}=\mathrm{C}$ double bonds which connect simple diamondoids, such as adamantane (Ada) or diamantane (Dia). Specifically, we consider Ada $=$ Ada, $\mathrm{Ada}=$ Dia, $\mathrm{Dia}=$ Dia and Ada $=$ Dia $=$ Ada, which are experimentally known. ${ }^{11}$ Dia $=$ Dia exists as two geometric isomers, $E$ and $Z$, depending on the geometry around the $\mathrm{C}=\mathrm{C}$ bond connecting the two diamantane units and has been studied with analogous theoretical models in ref. 18. Most optical and electronic properties of $E$ and $Z$ are very similar and so are their energies. For $\mathrm{Ada}=$ Ada and Ada $=$ Dia no analogous isomers exist. Fig. 1 shows the optimized geometries in their ground electronic states for the two other diamondoids containing a single $\mathrm{C}=\mathrm{C}$ bond as studied here, namely Ada $=$ Ada and $\mathrm{Ada}=$ Dia.

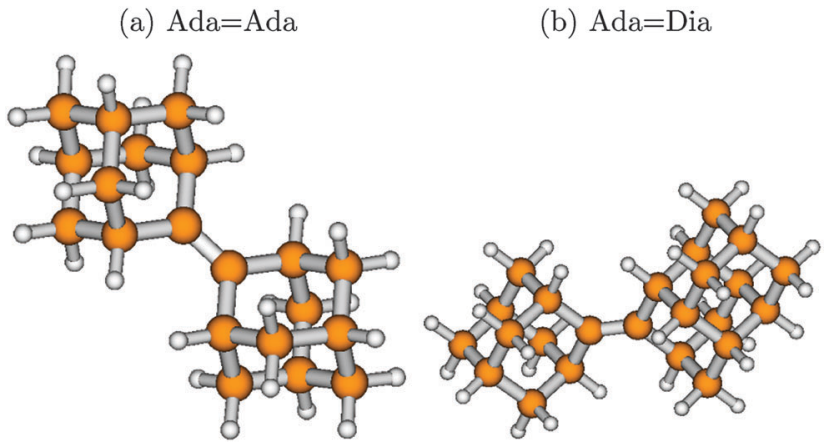

Fig. 1 The optimized geometries at the ground electronic states $\left(S_{0}, g\right)$ of $\mathrm{Ada}=\mathrm{Ada}(\mathrm{a})$ and Ada=Dia (b), at the B3LYP/TZVP level of theory. Brown balls represent the $\mathrm{C}$ atoms and the white balls $\mathrm{H}$ atoms. The central bond joining the two diamondoid units is a $\mathrm{C}=\mathrm{C}$ double bond, with a bond length of $1.34 \AA$ for either. Other $\mathrm{C}-\mathrm{C}$ bonds are single, with bond lengths typically of $1.54 \AA$. The $\mathrm{C}=\mathrm{C}$ bond-length compares well with recently available experimental (1.34 $\AA$ for Ada=Ada and $1.33 \AA$ for Ada=Dia) and theoretical (DFT and MP2) data. ${ }^{36}$ The calculated $\mathrm{C}-\mathrm{H}$ bond lengths $(1.09 \AA)$ also show good agreement with the corresponding experimental data.

Table 1 Values of HOMO-LUMO gap $\Delta E_{\mathrm{HL}}, 0-0$ transition energy $\Delta E_{0-0}$, vertical transition energy $\Delta E_{\text {vert }}$ and main maximum of the vibronic

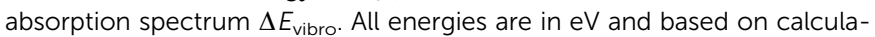
tions at B3LYP/TZVP level of theory, with the IMDHOFAD model used for $\Delta E_{0-0}$ and $\Delta E_{\text {vibro. }}$ Values for $\mathrm{Dia}=\mathrm{Dia}(E)$ and $\mathrm{Dia}=\mathrm{Dia}(Z)$ were already reported in ref. 18 . Also values for Ada are shown, taken from the same reference

\begin{tabular}{lllll}
\hline Molecule & $\Delta E_{\mathrm{HL}}$ & $\Delta E_{0-0}$ & $\Delta E_{\text {vert }}$ & $\Delta E_{\text {vibro }}$ \\
\hline Adamantane (Ada) & 8.15 & 6.54 & 7.32 & 6.84 \\
Ada $=$ Ada & 6.34 & 5.20 & 5.60 & 5.48 \\
Ada $=\operatorname{Dia}$ & 6.28 & 5.16 & 5.55 & 5.44 \\
Dia $=\operatorname{Dia}(E)$ & 6.23 & 5.14 & 5.52 & 5.41 \\
Dia $=\operatorname{Dia}(Z)$ & 6.22 & 5.11 & 5.50 & 5.38
\end{tabular}

Considering the lowest-energy, bright $\left(\mathrm{S}_{0} \rightarrow \mathrm{S}_{1}\right)$ absorption transitions of Dia $=$ Dia, Ada $=$ Ada and Ada $=$ Dia, we show in Table 1 the HOMO-LUMO gaps $\Delta E_{\mathrm{HL}}$ (calculated for Kohn-Sham orbital energies) and vertical excitation energies $\Delta E_{\text {vert }}$ (calculated from TD-DFT) for these compounds. We also compare to the lowest-energy, bright transitions for adamantane. ${ }^{18}$

Considering Ada as a reference first, experimentally it is known that this parent compound has an optical gap (the onset of absorption) of $6.49 \mathrm{eV},{ }^{10}$ which is close to the experimental 0-0 transition in this case (see also Table 6 below). (The optical gap of Dia is found to be $6.40 \mathrm{eV}$ according to that ref. 10.) From Table 1 we see that the theoretical $\Delta E_{0-0}$ value is close to the experimental one. The vertical excitation energy is $7.32 \mathrm{eV}$ for adamantane and unknown experimentally. From the table it is seen that the energy gaps decrease considerably when Ada/Dia subunits are connected by $\mathrm{C}=\mathrm{C}$ bonds. Taking $\Delta E_{0-0}$ values as a measure for optical gap reduction, the latter is in the order of $1.3 \mathrm{eV}$ and close to up to about $2 \mathrm{eV}$ if $\Delta E_{\mathrm{vert}}$ are considered (relative to Ada). Both the $\Delta E_{0-0}$ and $\Delta E_{\text {vert }}$ values of Ada $=$ Ada, Dia $=$ Dia and Ada $=$ Dia are all very similar (within $\sim 0.1 \mathrm{eV}$ ). The lowest allowed electronic transition to $\mathrm{S}_{1}$ for the electronically blended diamondoids involves a dominant HOMO $\rightarrow$ LUMO 
transition, which is relatively weak for Ada $=\mathrm{Ada}, \mathrm{Ada}=$ Dia and also the previously studied $\operatorname{Dia}=\operatorname{Dia}(E)$. For $\operatorname{Dia}=\operatorname{Dia}(E)$ the vertical transition energy to $S_{1}$ at $\omega_{1}=5.52 \mathrm{eV}$ has an oscillator strength of $f_{1}=0.00039 .{ }^{18}$ The HOMO is the $\mathrm{C}=\mathrm{C} \pi$-orbital, and the LUMO is a diffuse orbital delocalized over the periphery of the molecule. A similar behavior is found for the species studied here, $\mathrm{Ada}=\mathrm{Ada}$ and $\mathrm{Ada}=$ Dia. It should be noted that, due to relatively low oscillator strengths, the $\mathrm{S}_{1}$ state does not dominate the spectrum, however. Rather, the most intense transition is due to a $\mathrm{C}=\mathrm{C} \pi \rightarrow \pi^{*}$ excitation at higher energies. For $\operatorname{Dia}=\operatorname{Dia}(E)$, for example, $\pi^{*}$ is the LUMO +2 , which, like $\pi$, is strongly localized around the $\mathrm{C}=\mathrm{C}$ bond. The corresponding excited state is $\mathrm{S}_{4}$ in case of $\mathrm{Dia}=\operatorname{Dia}(E)$, at a vertical transition energy of $\omega_{4}=6.34 \mathrm{eV}$ and an oscillator strength of $f_{4}=0.8327 .^{18}$ (States $\mathrm{S}_{2}$ and $\mathrm{S}_{3}$ are dark states in case of Dia $=\operatorname{Dia}(E)$.)

Table 1 also demonstrates that $\Delta E_{\mathrm{HL}}$ values should not be considered as reliable estimates of optical gaps, being about $0.8 \mathrm{eV}$ larger than $\Delta E_{\mathrm{vert}}$. Another measure for optical excitations is the vibronic energy difference $\Delta E_{\text {vibro, }}$,.e., the maximum of the vibronically resolved absorption spectra. $\Delta E_{\text {vibro }}$ is also listed in the table, showing similar (but slightly lower) values than $\Delta E_{\text {vert }}$.
For the unsaturated species, $\Delta E_{\text {vert }}$ is reduced by about $1.8 \mathrm{eV}$ compared to a saturated diamondoid such as Ada. The strong reduction of the optical gap in $\mathrm{C}=\mathrm{C}$-connected dimers by the here observed order of magnitude is very consistent with experimental findings. ${ }^{11}$

To illustrate effects of the vibronic finestructure on spectra in detail, we show in Fig. 2 absorption, emission and resonance Raman spectra for Ada $=$ Ada and $\mathrm{Ada}=$ Dia, obtained by using (TD-)B3LYP/TZVP/IMDHOFAD and the $\mathrm{S}_{0}$ and $\mathrm{S}_{1}$ states. Let us consider the absorption and emission spectra (upper panels) first. For both molecules it is seen that indeed, the 0-0 transitions are not clearly visible either in absorption or in emission. This is because some modes exhibit very high values of adimensional shifts, up to 4 (compared to the usual values which lie around 1). All absorption and emission spectra show a pronounced vibrational finestructure. For both molecules, the maximal absorption peak is close to the vertical excitation energies, i.e., $\Delta E_{\mathrm{vert}} \sim$ $\Delta E_{\text {vibr }}$ as already demonstrated in Table 1 (both $\Delta E_{0-0}$ and $\Delta E_{\text {vert }}$ are shown as dashed, vertical lines). As a fine detail, we note that the absorption and emission spectra of Ada $=$ Dia (b) are slightly more structured than those of Ada $=$ Ada (a), but are (a) Ada=Ada
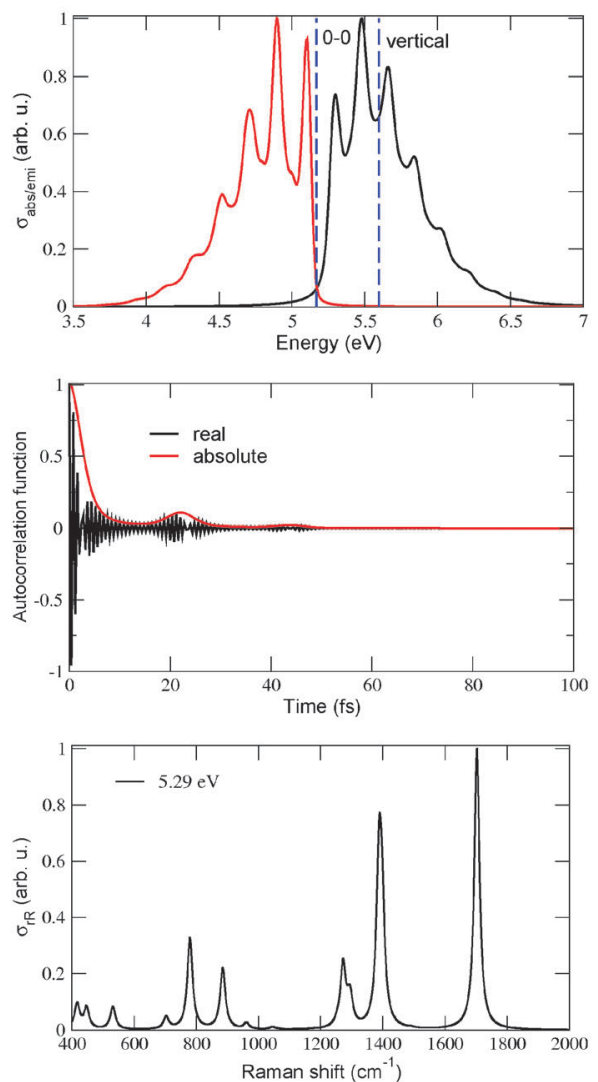

(b) Ada=Dia
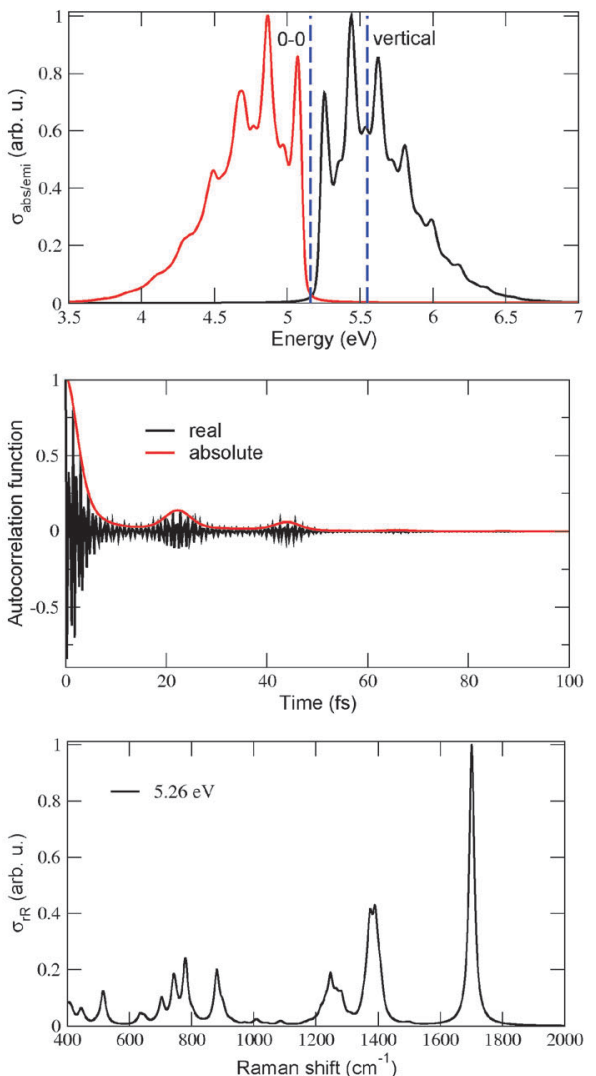

Fig. 2 The vibrationally resolved absorption, emission (upper panels) and $r R$ spectra (lowest panels) for Ada $=A d a(a)$ and Ada $=$ Dia (b), respectively. Vertical dashed lines in upper panels stand for $0-0$ and vertical (for absorption) transitions, respectively. Middle panels show the corresponding autocorrelation functions for absorption. For absorption and emission, damping factors $\Gamma=50 \mathrm{~cm}^{-1}$ have been used. Resonance Raman spectra in the lowest panels are for selected excitation energies $\omega_{\mathrm{L}}$ close to resonance. For these, a broadening factor $\tilde{\Gamma}=50 \mathrm{~cm}^{-1}$ has been used and the resulting stick spectrum was broadened by normalized Lorentzians of FWHM of $10 \mathrm{~cm}^{-1}$. All calculations were at the (TD-)B3LYP/TZVP/IMDHOFAD level of theory. Here and everywhere, spectra are normalized to 1 (hence "arbitrary units") for their highest peaks. 
similar otherwise. Also the corresponding spectra of Dia $=$ Dia (see Fig. 10 of ref. 18) look similar.

Vibrational peak spacings in absorption spectra approximately correspond to excited state vibrational frequencies of normal modes which are dominantly excited upon electronic excitation. (Similarly, peak spacings in emission spectra reflect dominant modes in the electronic ground state, after de-excitation.) For Ada $=$ Ada and $\mathrm{Ada}=$ Dia, it is the $\mathrm{C}=\mathrm{C}$ stretching mode which is the principal contributor to the vibrational finestructure in absorption. This is a similar feature as observed previously for $\mathrm{Dia}=\mathrm{Dia}^{18}$ and can be attributed to the location of the HOMO on the $\mathrm{C}=\mathrm{C}$ double bond connecting the two diamondoid units to each other. As reported for Dia $=$ Dia in ref. 18 , the $\mathrm{C}=\mathrm{C}$ bond lengths of $\mathrm{Ada}=$ Ada and Ada $=$ Dia also elongate by $0.06-0.07 \AA$ in the $\mathrm{S}_{1}$ excited state involving a HOMO $\rightarrow$ LUMO transition. At the same time, the vibrational frequency of the $\mathrm{C}=\mathrm{C}$ vibrations soften from about $1700 \mathrm{~cm}^{-1}$, to about $1530 \mathrm{~cm}^{-1} .^{18}$ Fig. 2(a) and (b), middle panel, show the absorption autocorrelation functions for $\mathrm{Ada}=\mathrm{Ada}$ and $\mathrm{Ada}=$ Dia, respectively. The most striking features are recurrences after about $22 \mathrm{fs}$ in both cases, which translate into a vibrational level spacing of about $1520 \mathrm{~cm}^{-1}$ (or about $0.19 \mathrm{eV}$ ) - in good correspondence to the softened $\mathrm{C}=\mathrm{C}$ vibration, also known from experiment. ${ }^{11}$ The slightly more pronounced recurrence features in Ada $=$ Dia compared to Ada $=$ Ada, explain the slightly more pronounced vibrational features in the spectra of the former.

To put our calculations in a wider context, we note that theoretically ${ }^{10,18}$ and also experimentally ${ }^{10}$ for Ada, as a reference, the lowest absorption band is highly vibrationally structured. Experimentally, the intensity of the first $\left(S_{1}\right)$ absorption band increases at least up to about $7 \mathrm{eV} \cdot{ }^{10}$ Experimentally, also the emission spectrum is highly finestructured, peaking at around $5.9 \mathrm{eV}^{10}$ In ref. 18, we found a highly structured fluorescence spectrum for Ada as well, with a maximum at around $6 \mathrm{eV}$. This and the absorption behavior found in ref. 11 gives us some confidence that the present trends in vibronic spectra for unsaturated diamondoids are reliable.

Concerning the rR spectra in Fig. 2, lowest panels, reported at excitation energies of $5.29 \mathrm{eV}$ (a) and $5.26 \mathrm{eV}$ (b), respectively, we note that also for these the $\mathrm{C}=\mathrm{C}$ stretching mode (now for the ground state with a frequency around $1700 \mathrm{~cm}^{-1}$ ) is the dominant scatterer. The excitation energies were chosen slightly above the corresponding $\Delta E_{0-0}$ values ( $c f$. Table 1 ) to establish a resonance effect. Some $\mathrm{C}-\mathrm{H}$ and $-\mathrm{CH}_{2}$ bending modes around 1300-1400 $\mathrm{cm}^{-1}$ and some low frequency $\mathrm{C}-\mathrm{C}-\mathrm{C}$ torsional modes are also intense. Again, the general behavior is similar to $\mathrm{Dia}=\mathrm{Dia}^{18}$ and in good agreement with experimental data for $\mathrm{C}=$ C-connected diamondoids. ${ }^{11}$

Another system which will consider is $\mathrm{Ada}=\mathrm{Dia}=\mathrm{Ada}$. This molecule is particularly interesting because of the presence of two $\mathrm{C}=\mathrm{C}$ double bonds and also because of methodological interest. An attempt to obtain an optimized equilibrium geometry (on the B3LYP/TZVP level) for the $S_{1}$ state of Ada $=$ Dia $=$ Ada was not successful. However, on changing the basis set to a less accurate $6-31 \mathrm{G}^{*}$ basis set, again using the B3LYP functional, an optimized $\mathrm{S}_{1}$ state was obtained, whose equilibrium geometry (a)

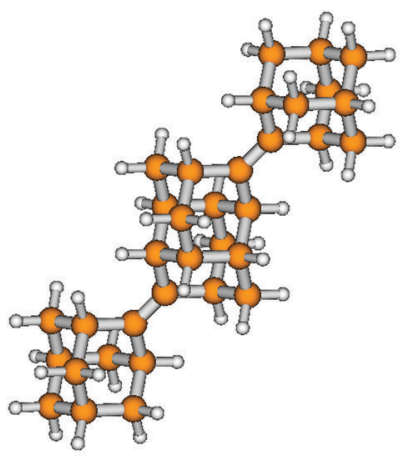

(b)

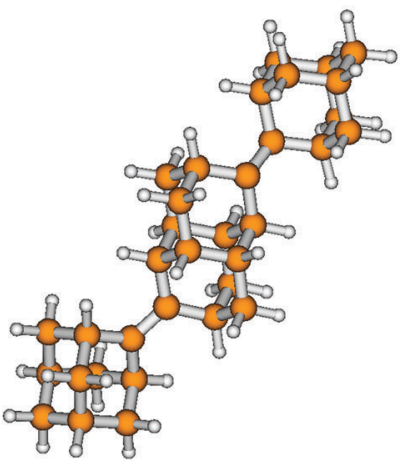

Fig. 3 The minimum geometries of the (a) $S_{0}$ and (b) $S_{1}$ electronic states of $\mathrm{Ada}=\mathrm{Dia}=\mathrm{Ada}$, calculated at the B3LYP/6-31G* level of theory. In the $\mathrm{S}_{1}$ state, the adamantane unit in the extreme right undergoes a rotation about the $\mathrm{C}=\mathrm{C}$ bond joining it to the adjacent diamantane unit.

was confirmed by only real frequencies in the subsequent normal mode analysis. Fig. 3 shows the optimized geometries of the $S_{0}$ and $S_{1}$ states at the B3LYP/6-31G* level of theory. It can be seen that during the $\mathrm{S}_{0} \rightarrow \mathrm{S}_{1}$ transition, a torsion of one of the rings around one of the $\mathrm{C}=\mathrm{C}$ bonds occurs. This $\mathrm{C}=\mathrm{C}$ bond is elongated to $1.45 \AA$ in the $S_{1}$ state, compared to its value of $1.35 \AA$ in the ground state, hence facilitating the rotation of one of the diamondoid units around itself. There were slight rotations of some of the other cyclohexane rings as well.

One of the main effects of these rotations is that, many of the normal modes have very high values of dimensionless origin shifts, resulting in unphysically low vibronic overlap and a broad unphysical absorption spectrum. This is a case where the IMDHO (STA) approach as a method based on a "vertical" model is expected to be more realistic. Consequently, this method was used to obtain vibronic spectra.

The absorption and rR spectra obtained on the B3LYP/ 6-31G* level, using the IMDHO (STA) model are shown in Fig. 4(a) and (b) respectively.

The general characteristics of the absorption and rR spectra are similar to those of the electronically blended diamondoids described earlier. The contributors of the vibrational spacings in the absorption spectrum and the principal Raman scatterer in the rR spectrum are mainly the nearly degenerate stretching modes of the two $\mathrm{C}=\mathrm{C}$ bonds, with ground state vibrational frequencies around $1700 \mathrm{~cm}^{-1}$ according to our B3LYP/TZVP calculations.

Compared to Ada $=$ Ada and Ada $=$ Dia (Fig. 2), for $\mathrm{Ada}=\mathrm{Dia}=$ Ada the absorption range is blue-shifted again, by about $0.5-0.6 \mathrm{eV}$. In the calculation this comes about by the fact that the adiabatic minima separation energy between $S_{0}$ and $S_{1}$ in the IMDHO (STA) approach does not involve a full optimization of the $\mathrm{S}_{1}$ state. The "Frank-Condon-extrapolated" $\mathrm{S}_{1}$ state is $0.4 \mathrm{eV}$ higher in energy than what one finds for comparable systems, for which a full excited state geometry optimization was carried out. This is demonstrated in Table 2, where we compare adiabatic minima separation energy values for Dia $=$ Dia $(\mathrm{B} 3 \mathrm{LYP} / \mathrm{TZVP}), \mathrm{Ada}=\mathrm{Dia}=\mathrm{Ada}\left(\mathrm{B} 3 \mathrm{LYP} / 6-31 \mathrm{G}^{*}\right)$ and $\mathrm{Ada}=\mathrm{Dia}=\mathrm{Ada}\left(\mathrm{B} 3 \mathrm{LYP} / 6-31 \mathrm{G}^{*}, \mathrm{STA}\right)$. Note that for Ada $=\mathrm{Dia}=$ Ada 
(a)

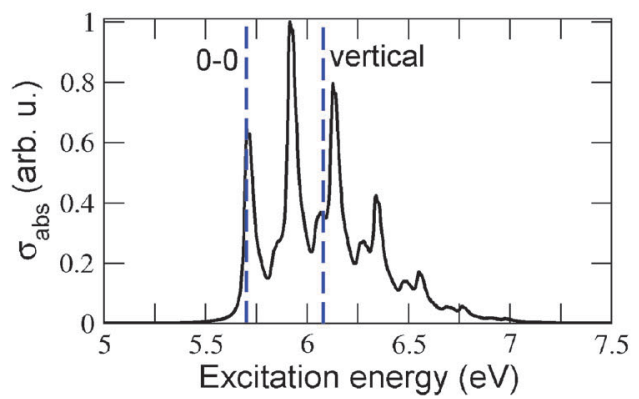

(b)

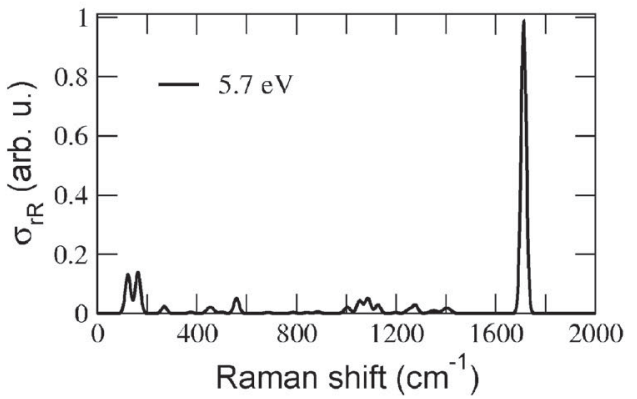

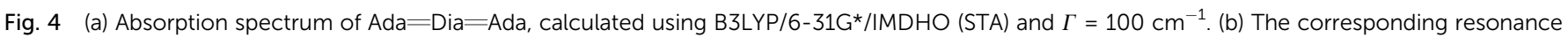

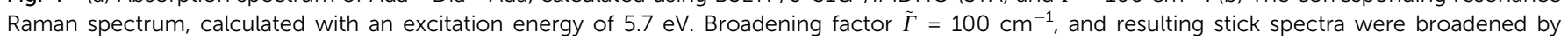
Lorentzians of FWHM $10 \mathrm{~cm}^{-1}$.

Table 2 Values of the adiabatic minima separation energy between $\mathrm{S}_{0}$ and $\mathrm{S}_{1}$ as obtained for $\mathrm{Dia}=\mathrm{Dia}(E)$ and $\mathrm{Ada}=\mathrm{Dia}=\mathrm{Ada}$ using the different approaches mentioned

\begin{tabular}{lll}
\hline Molecule & Method & $E_{0}(\mathrm{eV})$ \\
\hline $\mathrm{Dia}=\mathrm{Dia}(E)$ & B3LYP/TZVP/IMDHOFAD & 5.27 \\
$\mathrm{Ada}=\mathrm{Dia}=$ Ada & B3LYP/TZVP/IMDHOFAD & 5.28 \\
$\mathrm{Ada}=\mathrm{Dia}=$ Ada & B3LYP/TZVP/STA & 5.70
\end{tabular}

(B3LYP/6-31G*/IMDHOFAD) and $\mathrm{Dia}=\mathrm{Dia}(E) \quad(\mathrm{B} 3 \mathrm{LYP} / \mathrm{TZVP} /$ IMDHOFAD) the fully optimized adiabatic energy differences are very similar, in contrast to Ada $=$ Dia $=$ Ada $(\mathrm{B} 3 \mathrm{LYP} / \mathrm{TZVP} /$ STA), which gives the mentioned higher value. The question arises then if indeed, the blue-shifted absorption spectrum (and also the rR spectrum) shown in Fig. 4 are realistic, or numerical artefacts. An experiment could be of great value here.

\section{B. Functionalized diamondoids: thiols, thiones and related species}

We now turn to the second way of modifying diamondoids, by introducing ligands. We shall mostly focus on sulfur-containing materials, thiols and thiones, but also touch diamondoids functionalized with alcohol $(-\mathrm{OH})$ and bromine $(-\mathrm{Br})$ groups. For simplicity, as a parent compound only adamantane will be considered.

1. Thiols. The ground electronic states of (adamantane-) 1thiol, 2-thiol, 1,2-dithiol, 1,3-dithiol, 2,4-dithiol, 2,6-dithiol and 2,7-dithiol were optimized at the B3LYP/TZVP level of theory. The goal here was to study (i) effects of the positioning of the SH group(s) and (ii) effects of multi-substitution. The optimized geometries of some of the thiols are shown in Fig. 5. The nomenclature used can also be understood from this figure.

The $S_{1}$ state is the first bright excited electronic state, resulting from a dominant HOMO $\rightarrow$ LUMO excitation. For adamantane-1-thiol, for example, the HOMO has maximum contribution from the non-bonding orbital of sulphur and partial contribution from the $\mathrm{C}-\mathrm{C}$ bonds. The LUMO is partially delocalized towards the outer periphery, much like that of adamantane, but also has contribution from anti-bonding orbitals of the $\mathrm{S}-\mathrm{H}$ bond, consistent with previous calculations at the CC2/6-311++G** level of theory. ${ }^{16}$ As a consequence, the

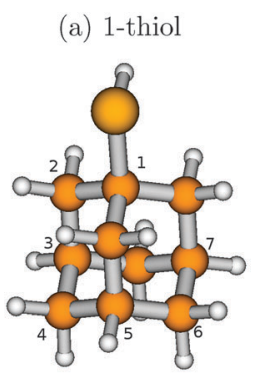

(b) 2-thiol

(c) 1,2-dithiol
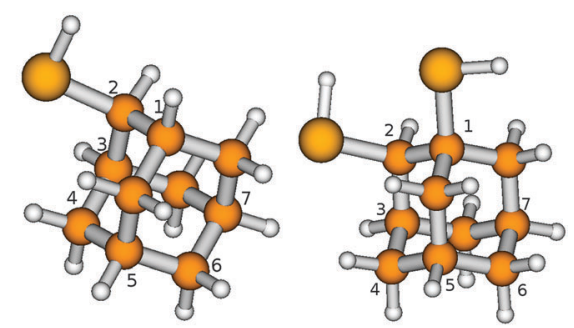

Fig. 5 The geometries of the optimized $\mathrm{S}_{0}$ states of three isomers of adamantane-1-thiol (a) and adamantane-2-thiol (b), both $\mathrm{C}_{10} \mathrm{H}_{15} \mathrm{SH}$, as well as adamantane-1,2-dithiol (c), $\mathrm{C}_{10} \mathrm{H}_{14}(\mathrm{SH})_{2}$, obtained at the B3LYP/TZVP level of theory. The large yellow balls represent the $\mathrm{S}$ atoms, while the smaller brown balls and the white balls represent the $C$ and $\mathrm{H}$ atoms respectively.

$\mathrm{S}_{1}$ states of the thiols could not be optimized, because of the high lability of the $\mathrm{S}-\mathrm{H}$ bond which dissociated during the optimization process. Still, $0-0$ transition energies $\Delta E_{0-0}$ and vibronic transition energies $\Delta E_{\text {vert }}$ can be calculated from the IMDHO (STA) approach. Table 3 summarizes the HOMOLUMO energy gaps, the 0-0 transition energies, the vertical transition energies and the absorption maxima for the $S_{0} \rightarrow S_{1}$ excitation of the thiols as obtained in this way. Comparison with the parent molecule, adamantane, is also provided (data taken from ref. 18 and with $\Delta E_{0-0}$ and $\Delta E_{\text {vert }}$ obtained with the B3LYP/TZVP/IMDHOFAD model, though).

It is seen from Table 3 that the vertical excitation energies $\Delta E_{\text {vert }}$ decrease with increasing thiol substitution. The $\Delta E_{\text {vert }}$ values change by about $2 \mathrm{eV}$ when a single $-\mathrm{SH}$ group is introduced, nearly independent of position (1 or 2). For the dithiols, the vertical excitation energies do either decrease only slightly (for 2,6- and 2,7-dithiols), or show a slight additional redshift (of up to about $0.15 \mathrm{eV}$, for 1,2-dithiol), relative to monothiols. The extra redshift is found to be the larger, the farther apart the two thiol groups are. This is also found for the $\Delta E_{0-0}$ and $\Delta E_{\text {vibro }}$ values, while the (less reliable) HOMO-LUMO gaps suggest otherwise.

Vibrationally resolved absorption and emission spectra of these thiols and dithiols, were also calculated using the IMDHO 
Table 3 The HOMO-LUMO gap $\Delta E_{\mathrm{HL}}, 0-0$ transition energy $\Delta E_{0-0}$, vertical transition energy $\Delta E_{\text {vert }}$ and main maximum of the vibronic absorption spectrum $\Delta E_{\text {vibro }}$ for some selected thiols and dithiols of adamantane, calculated at the B3LYP/TZVP level of theory, for $\mathrm{S}_{0} \rightarrow \mathrm{S}_{1}$ excitations. The $\Delta E_{0-0}$ and $\Delta E_{\text {vibro }}$ values correspond to the IMDHO (STA) model. The corresponding values for adamantane ( $\Delta E_{0-0}$ and $\Delta E_{\text {vibro }}$ using the IMDHOFAD model) have also been provided to show the fall of the optical gap with functionalization. All reported values of energy are in $\mathrm{eV}$

\begin{tabular}{lllll}
\hline Molecule & $\Delta E_{\mathrm{HL}}$ & $\Delta E_{0-0}$ & $\Delta E_{\mathrm{vert}}$ & $\Delta E_{\mathrm{vibro}}$ \\
\hline Adamantane & 8.15 & 6.54 & 7.32 & 6.84 \\
1-Thiol & 6.46 & 4.77 & 5.29 & 5.15 \\
2-Thiol & 6.50 & 4.78 & 5.31 & 5.14 \\
& & & & \\
1,2-Dithiol & 6.20 & 4.07 & 5.14 & 5.06 \\
1,3-Dithiol & 6.29 & 4.89 & 5.24 & 5.04 \\
2,4-Dithiol & 6.25 & 4.66 & 5.22 & 5.11 \\
2,6-Dithiol & 6.36 & 5.04 & 5.30 & 5.16 \\
2,7-Dithiol & 6.41 & 4.98 & 5.29 & 5.11
\end{tabular}

(STA) model at the B3LYP/TZVP level of theory. A full excited state $\left(S_{1}\right)$ geometry optimization was not possible in most cases for the reasons stated above and therefore showing these $\mathrm{S}_{0} \leftrightarrow \mathrm{S}_{1}$ vibronic spectra is of little value here. It is sufficient to say that for several molecules (adamantane-2-thiol, 1,2- and 2,4-dithiol) a general trend is the absence of well-resolved vibrational finestructure, due to large normal mode displacements. For others (in particular 2,6- and 2,7-dithiol), a clear vibrational finestructure is seen both in IMDHO (STA) absorption and emission. We also mention that according to this approach, emission spectra for thiols can be strongly redshifted, in particular for 1,2-dithiol, whose emission spectrum peaks around $\sim 3 \mathrm{eV}(\sim 400 \mathrm{~nm})$.

The $S_{0} \leftrightarrow S_{1}$ state pair gives interesting trends but may not be very relevant for optical properties of thiolated diamondoids, however. In fact, in ref. 37 Landt and co-workers measured the absorption and fluorescence spectra of adamantane-1-thiol. The optical gap was measured to be $5.85 \mathrm{eV}$, i.e. redshifted by $0.64 \mathrm{eV}$ relative to adamantane $(6.49 \mathrm{eV}$, see above and also Table 6 below).$^{37}$ It was also mentioned in ref. 37 that probably an extremely weak, dissociative transition occurs below $5.5 \mathrm{eV}$, whose low intensity is outside the reach of optical gap measurements, however. We believe that this weak transition is the $\mathrm{S}_{0}$ to $\mathrm{S}_{1}$ transition reported above (with $\Delta E_{\text {vert }}=5.29 \mathrm{eV}$ and $\Delta E_{0-0}=4.77 \mathrm{eV}$ according to Table 3). Being a dissociative state, it is believed that this weak $S_{1}$ state of the thiol does not fluoresce; in fact no fluorescence has been observed for any of the diamondoid thiols. $^{37}$

For 1-thiol, TD-B3LYP/TZVP calculations showed two other interesting states, $S_{2}$ and $S_{3}$ states with $\Delta E_{\text {vert }}$ values of $6.32 \mathrm{eV}$ and $6.67 \mathrm{eV}$, respectively. Both are optically allowed, however, the $\mathrm{S}_{0} \rightarrow \mathrm{S}_{2}$ transition only weakly in contrast to $\mathrm{S}_{0} \rightarrow \mathrm{S}_{3}$ (the oscillator strengths being $f_{2}=0.0005$ and $f_{3}=0.0088$, respectively). Geometry optimization and normal mode analysis was possible and performed, for the $\mathrm{S}_{3}$ state. Comparison with the $\mathrm{S}_{1}$ data revealed an important difference in $\mathrm{S}_{3}$ : the S-H bondlength remained essentially unaltered compared to the ground state, whereas the $\mathrm{C}-\mathrm{S}$ bond length increased from $1.86 \AA$ to $1.99 \AA$. The $\Delta E_{0-0}$ value on the TD-B3LYP/TZVP/IMDHOFAD level of theory, was found to be $6.32 \mathrm{eV}$ for $\mathrm{S}_{3}$, somewhat blueshifted compared to the measured optical gap. The $S_{3}$ state is redshifted with respect to (the $S_{1}$ state) of adamantane, however, by $0.65 \mathrm{eV}$ when $\Delta E_{\text {vert }}$ values are taken as a reference and by $0.22 \mathrm{eV}$ if $\Delta E_{0-0}$ values are considered.

With TD-B3LYP/TZVP/IMDHOFAD we also calculated vibrationally resolved absorption, emission and $\mathrm{rR}$ spectra for the $\mathrm{S}_{0} \leftrightarrow \mathrm{S}_{3}$ state pair (cf. Fig. 6). The absorption spectrum (Fig. 6(a)) shows considerable vibrational finestructure. The $\Delta E_{\text {vibro }}$ value is $6.32 \mathrm{eV}$ and thus equal $\Delta E_{0-0}$, because in this case the $0-0$ transition is the most intense in the vibronically resolved absorption spectrum. (One must say, though, that the peak intensity depends on the broadening factor $\Gamma$ and hence also $\Delta E_{\text {vibro }}$ may shift somewhat with other $\Gamma$ values.) When $\mathrm{S}_{3}$ is considered as "the" most relevant low-energy excitation of adamantane-1-thiol, then also for the photoluminescence (emission) spectrum a strong vibrational progression is predicted (Fig. 6(a)). The center of the emission spectrum $S_{3} \rightarrow S_{0}$ is slightly above $6 \mathrm{eV}$, i.e., certainly not as low as predicted for $\mathrm{S}_{1} \rightarrow \mathrm{S}_{0}$ (where the center was found at $\sim 4.5 \mathrm{eV}$ within the B3LYP/TZVP/IMDHO (STA) model, not shown).

The resonance Raman spectrum, computed at an excitation energy $\omega_{\mathrm{L}}=6.43 \mathrm{eV}$ and shown in Fig. 6(b) reveals as a dominant scatterer, the $\mathrm{C}-\mathrm{S}$ stretching mode at $1060 \mathrm{~cm}^{-1}$. This is quite expected from the considerable enhancement of (a)

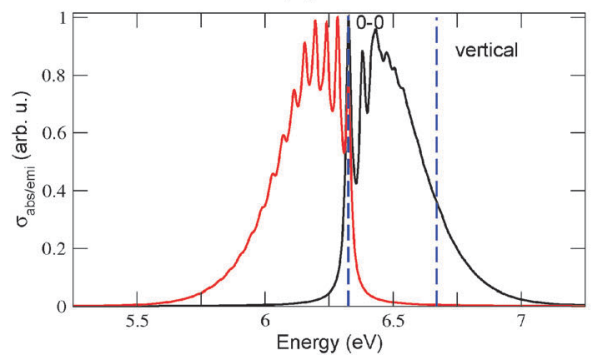

(b)

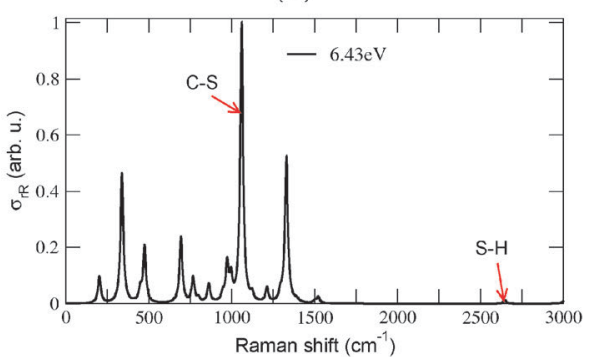

Fig. 6 (a) Absorption and emission spectra of adamantane-1-thiol, calculated using B3LYP/TZVP/IMDHOFAD and $\Gamma=100 \mathrm{~cm}^{-1}$. (b) The corresponding resonance Raman spectrum, calculated with an excitation energy of $6.43 \mathrm{eV}$. Broadening factor $\tilde{\Gamma}=100 \mathrm{~cm}^{-1}$, and resulting stick spectra were broadened by Lorentzians of FWHM $10 \mathrm{~cm}^{-1}$. Two relevant peaks are indicted by arrows (see text). 
the $\mathrm{C}-\mathrm{S}$ bond length in the $\mathrm{S}_{3}$ excited state. The rR spectrum of adamantane-1-thiol in Fig. 6(b) is in contrast to the one obtained when using the IMDHO (STA) model and the $\mathrm{S}_{1}$ state as the resonant state. The corresponding rR spectrum (not shown) predicts as by far the most intense scatterer, an $\mathrm{S}-\mathrm{H}$ stretching vibration at $2650 \mathrm{~cm}^{-1}$. (The same is observed for almost all thiols studied in this work, when using the $S_{0} \leftrightarrow S_{1}$ IMDHO (STA) model.) According to Fig. 6(b), however, the S-H vibration has only very little intensity in the $S_{0} \leftrightarrow S_{3}$ IMDHOFAD model. We thus suggest that $\mathrm{rR}$ spectroscopy may be a valuable tool to judge on the "optical importance" of electronically excited states of thiols.

2. Thiones. In the thiones, the two hydrogen atoms in a methylene $\left(\mathrm{CH}_{2}\right)$ unit are replaced by sulfur (S). The B3LYP/TZVP optimized ground state geometries of adamantane-2-thione, adamantane-2,4-dithione and adamantane-2,6-dithione, which were studied in this work, are shown in Fig. 7.

Table 4 shows the vertical transition energies and the corresponding oscillator strengths for the $S_{0}$ to $S_{1}, S_{2}$ and $S_{3}$ transitions of the thiones studied in this work.

For adamantane-2-thione and adamantane-2,6-dithione, the $\mathrm{S}_{0} \rightarrow \mathrm{S}_{1}$ transitions are dipole forbidden. The first bright state for adamantane thione at the TD-B3LYP/TZVP level of theory is the $\mathrm{S}_{2}$ state (around $5.27 \mathrm{eV}$ ) which is dominated by a (HOMO-2) $\rightarrow$ LUMO transition. For the 2,6-dithione it is the $\mathrm{S}_{3}$ state $(3.34 \mathrm{eV}$, in the near-UV/visible range) which has contributions from (HOMO-1) $\rightarrow$ LUMO and HOMO $\rightarrow$ (LUMO+1) excitations. For adamantane-2,4-dithione, the $\mathrm{S}_{1}$ state at $2.39 \mathrm{eV}$ is a weakly allowed transition involving a HOMO $\rightarrow$ LUMO excitation. Note that the introduction of (more than one) thione groups lowers the optical gap considerably, into the visible regime, in agreement with findings of ref. 22. The HOMO for the thiones are predominantly non-bonding (n) orbitals located on $\mathrm{S}$, while the LUMO are anti-bonding $\pi^{*}$ orbitals centered on the $\mathrm{C}=\mathrm{S}$ double bond. The (HOMO-1), on the other hand, are the $\mathrm{C}=\mathrm{S} \pi$ bonding orbitals and the (HOMO-2) are $\sigma$ orbitals involving the $\mathrm{C}-\mathrm{H}$ and $\mathrm{C}-\mathrm{C}$ bonds of the cyclohexane rings. In particular the low-lying $\pi^{*} \mathrm{C}=\mathrm{S}$ orbitals cause the low-energy excitations in thiones.

Optimization of the respective first bright excited state was done at the TD-B3LYP/TZVP level. For 2,6-dithione it was not possible to obtain an $\mathrm{S}_{3}$ minimum. For adamantane-2-thione and adamantane-2,4-dithione it was possible to obtain $\mathrm{S}_{2}$ and

(a) 2-thione (b) 2,4-dithione
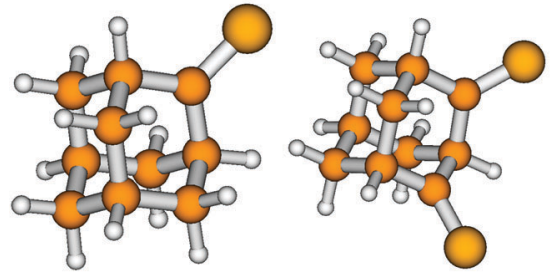

Fig. 7 The B3LYP/TZVP optimized ground state geometries of 2-thione (a), 2,4-dithione (b) and 2,6-dithione (c) of adamantane.
Table 4 In the upper half, the vertical transition energies and oscillator strengths for the first three excited states (labeled as $\alpha$ ) of adamantane-2thione, adamantane-2,4-dithione and adamantane-2,6-dithione. For adamantane-2-thione, $\mathrm{S}_{2}$ is dominated by a HOMO-2 $\rightarrow$ LUMO transition, for the 2,4-dithione, the $S_{1}$ is a HOMO $\rightarrow$ LUMO transition. For the 2,6-dithione, the $S_{3}$ involves a HOMO-1(HOMO) $\rightarrow$ LUMO(LUMO+1) transition. The lowest optically allowed transitions are shown in bold, and correspond to $\Delta E_{\mathrm{vert}}$ values in other tables. In the lower part of the table the $\Delta E_{0-0}, \Delta E_{\mathrm{HL}}$ and $\Delta E_{\text {vibro }}$ energies are given ( $\Delta E_{0-0}$ and $\Delta E_{\text {vibro }}$ for the lowest allowed transition). All calculations were done on the B3LYP/TZVP/IMDHO (STA) level of theory; all energies are in eV

\begin{tabular}{|c|c|c|c|c|c|c|}
\hline \multirow[b]{2}{*}{ State $\alpha$} & \multicolumn{2}{|c|}{ 2-Thione } & \multicolumn{2}{|c|}{ 2,4-Dithione } & \multicolumn{2}{|c|}{ 2,6-Dithione } \\
\hline & $\omega_{\alpha}(\mathrm{eV})$ & $f_{\alpha}$ & $\omega_{\alpha}(\mathrm{eV})$ & $f_{\alpha}$ & $\omega_{\alpha}(\mathrm{eV})$ & $f_{\alpha}$ \\
\hline 1 & 2.53 & 0.0000 & 2.39 & 0.0043 & 2.51 & 0.0000 \\
\hline 2 & 5.27 & 0.0065 & 2.51 & 0.0000 & 2.52 & 0.0000 \\
\hline 3 & 5.32 & 0.2291 & 3.24 & 0.0002 & 3.34 & 0.0008 \\
\hline$\Delta E_{0-0}$ & 4.99 & & 2.30 & & 3.17 & \\
\hline$\Delta E_{\mathrm{HL}}$ & 3.91 & & 3.51 & & 3.90 & \\
\hline$\Delta E_{\text {vibro }}$ & 5.17 & & 2.35 & & 3.17 & \\
\hline
\end{tabular}

$\mathrm{S}_{1}$ minima, respectively, and perform normal mode analyses in these states. Various parts of the molecules underwent significant distortion during the electronic excitation. In adamantane2-thione, one of the $\mathrm{C}-\mathrm{C}-\mathrm{C}$ bond angles contracts by $10-12^{\circ}$ in the excited state. In the 2,4-dithione, the $\mathrm{C}-\mathrm{C}-\mathrm{C}$ bond angle in between the two thione units (see Fig. 7) contracts by $17^{\circ}$ in the $\mathrm{S}_{3}$ state, bringing the two thione groups closer in space. Some of the $\mathrm{C}-\mathrm{C}-\mathrm{C}$ bond angles also increase by about $4-5^{\circ}$ in both molecules. For adamantane-2-thione, the $\mathrm{C}=\mathrm{S}$ bond length increases by $0.08 \AA(1.63 \AA \rightarrow 1.71 \AA)$ in the $\mathrm{S}_{2}$ state as compared to the $\mathrm{S}_{0}$ state, whereas in the 2,4-dithione, the two $\mathrm{C}=\mathrm{S}$ bond lengths increase by about $0.04 \AA$ in the $\mathrm{S}_{1}$ state. Due to several significant geometry changes, a number of normal modes, especially low frequency modes involving torsion of $\mathrm{C}-\mathrm{C}-\mathrm{C}$ units or bending vibrations of the $\mathrm{C}=\mathrm{S}$ bonds show very high values of dimensionless origin shifts between the ground and excited states. As a result, the IMDHOFAD approach produced broad, smooth spectra with no vibrational finestructure. Hence, similar to the procedure followed for the thiols, the vibronic absorption, emission and $\mathrm{rR}$ spectra of the three diamondoid thiones were calculated using the IMDHO (STA) approach implemented in ORCA. Fig. 8(a) shows the absorption and emission spectra of the three thiones calculated using the IMDHO (STA) approach at the B3LYP/TZVP level of theory.

The spectra of the three molecules are energetically shifted with respect to each other, because of the different excitation energies. The absorption and emission spectra of adamantane2,4-dithione lie in the visible energy range. The fluorescence emission spectrum of the 2,6-dithione also lies in the visible range, while the tail of the absorption spectrum extends just beyond the visible range. Adamantane-2-thione, on the other hand, absorbs and emits beyond the visible region. Vibronic finestructure is observed for adamantane-2-thione and the 2,6-dithione. The peak spacings in the absorption spectra are in the range of $520-530 \mathrm{~cm}^{-1}$ for adamantane-2-thione, whereas for the 2,6-dithione, the spacings lie in the range of $460-470 \mathrm{~cm}^{-1}$. 
(a)

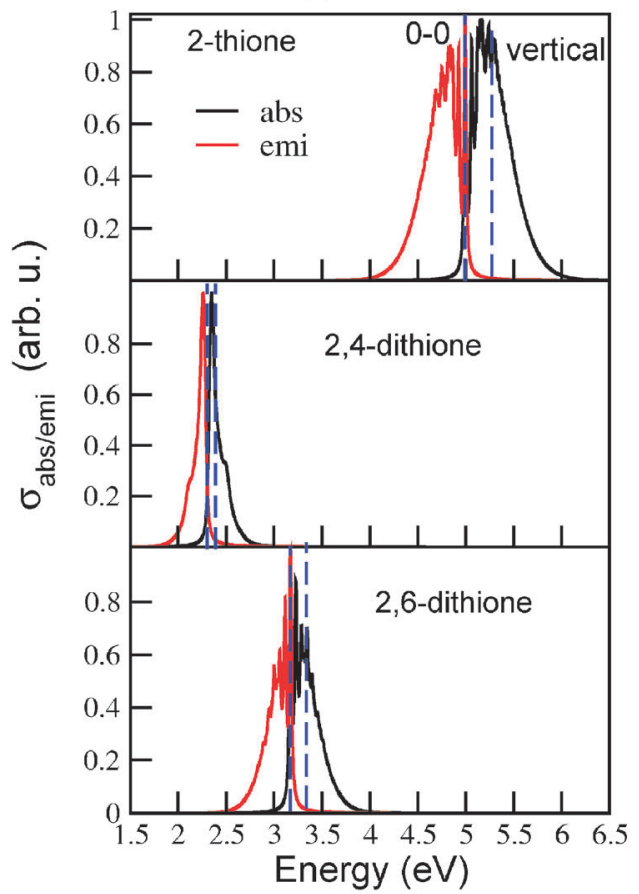

(b)

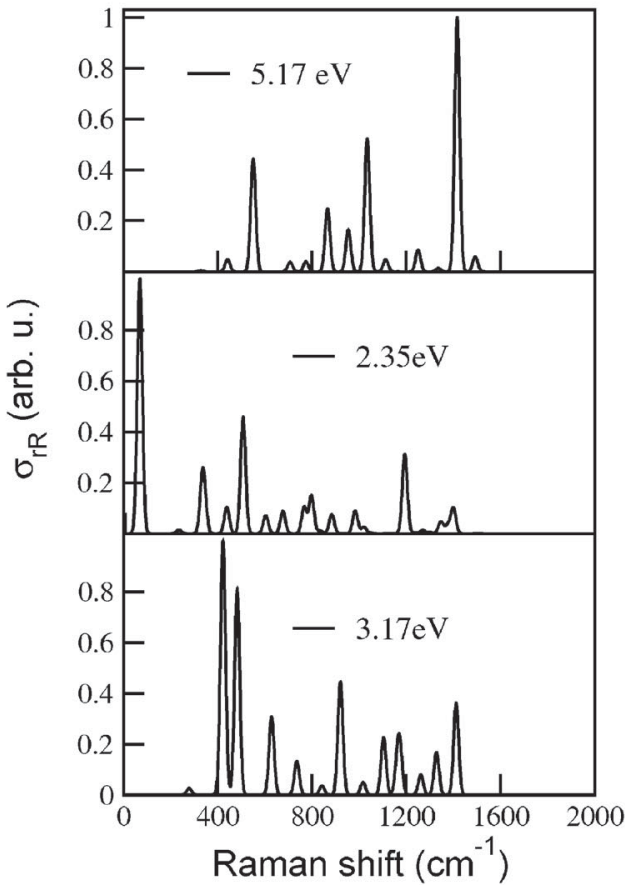

Fig. 8 (a) The vibronic absorption and emission spectra of the diamondoid thiones studied here, calculated at the B3LYP/TZVP level of theory, using the IMDHO (STA) model. From top to bottom the spectra are in the order: adamantane-2-thione, adamantane-2,4-dithione and adamantane-2,6-dithione. The dashed blue lines represent the $0-0$ and vertical transition energies for all three plots. (b) The corresponding rR spectra, using the same model. The excitation energies $\omega_{\mathrm{L}}$ are also indicated and correspond to $\Delta E_{\text {vibro }}$ of each molecule. Broadening parameter of $\Gamma$ and $\tilde{\Gamma}=100 \mathrm{~cm}^{-1}$ were used. For $\mathrm{rR}$, resulting stick spectra were broadened by Lorentzians with FWHM of $10 \mathrm{~cm}^{-1}$.

The absorption and emission spectra of 2,4-dithione show a broad peak and a shoulder. The values of the HOMO-LUMO gaps $\left(\Delta E_{\mathrm{HL}}\right), 0-0$ energies $\left(\Delta E_{0-0}\right)$, and maxima of the absorption spectra $\left(\Delta E_{\text {vibro }}\right)$ of the species are mentioned in Table 4 , lower part. (The corresponding $\Delta E_{\text {vert }}$ energies are shown in the upper half in bold). The IMDHO (STA) 0-0 energy (4.99 eV) of adamantane-2-thione is somewhat higher than a previously calculated value $(4.66 \mathrm{eV})$ of the absorption onset (the so-called zero-phonon line) of Demján and co-workers ${ }^{38}$ using the $G_{0} W_{0}$ quasiparticle energy-correction on solutions of the BetheSalpeter equations (BSE) ${ }^{39}$ based on LDA wavefunctions. The experimentally reported value of the absorption onset is approximately $4.50 \mathrm{eV} .^{40}$

Resonance Raman spectra (Fig. 8(b)) were calculated to get more insight into the normal modes facilitating the electronic transition. The spectra were calculated at excitation energies corresponding to $\Delta E_{\text {vibro }}$ of the individual molecules. The rR spectrum of adamantane-2-thione is dominated by a combination of the $\mathrm{C}=\mathrm{S}$ stretching mode and $\mathrm{C}-\mathrm{H}$ wagging modes, around $1420 \mathrm{~cm}^{-1}$. Lower frequency modes around $1030 \mathrm{~cm}^{-1}$ (characterized by $\mathrm{C}-\mathrm{C}-\mathrm{C}$ bending motions) and $550 \mathrm{~cm}^{-1}$ (characterized by $\mathrm{C}-\mathrm{S}$ stretching and $\mathrm{C}-\mathrm{C}-\mathrm{C}$ bending motions) also show considerable intensities. The involvement of $\mathrm{C}-\mathrm{S}$ stretching modes and $\mathrm{C}-\mathrm{C}-\mathrm{C}$ bending modes in $\mathrm{rR}$ scattering support the large increase of the $\mathrm{C}=\mathrm{S}$ bond length and change in $\mathrm{C}-\mathrm{C}-\mathrm{C}$ bond angles, upon electronic excitation, as mentioned earlier. The rR spectrum of the 2,4-dithione is mainly dominated by the first mode, around $67 \mathrm{~cm}^{-1}$, which is characterized by strong torsional motion of the $\mathrm{C}-\mathrm{C}-\mathrm{C}$ unit connecting the two thione groups. It is the same unit which contracted by $17^{\circ}$ in the excited state. One of the other important modes is the one around $1190 \mathrm{~cm}^{-1}$, involving stretching of the two $\mathrm{C}=\mathrm{S}$ bonds. For the 2,6-dithione, normal modes involving $\mathrm{C}-\mathrm{C}-\mathrm{C}$ torsion and $\mathrm{C}=\mathrm{S}$ stretch are also important scatterers.

3. Adamantane alcohols and bromo-adamantanes. Replacing the thiol $(-\mathrm{SH})$ groups in the adamantane thiols by alcohol $(-\mathrm{OH})$ or bromo $(-\mathrm{Br})$ groups we obtain adamantane alcohols and bromoadamantanes, respectively. We have studied 1- and 2-alcohols and bromo compounds in analogy to 1- and 2-thiols, as well as 1,2- and 2,6-dialcohols and dibromo adamantanes, in analogy to 1,2- and 2,6-dithiols. In Table 5 we list vertical excitation energies $\mathrm{S}_{0} \rightarrow \mathrm{S}_{1}$ for these alcohols and bromo-adamantanes, in comparison to the corresponding thiols, all obtained on the B3LYP/TZVP level of theory. All $S_{1}$ states are "bright".

Table 5 Vertical transition energy $\Delta E_{\text {vert }}$ for 1-adamantane-X, 2-adamantane- $X$ and 1,2-adamantane- $X$ and 2,6-adamantane- $X$, where $\mathrm{X}=\mathrm{SH}, \mathrm{OH}$ and $\mathrm{Br}$, obtained on the B3LYP/TZVP level of theory. All reported values of energies are in $\mathrm{eV}$

\begin{tabular}{lllll}
\hline$\Delta E_{\text {vert }}$ & $1-\mathrm{X}$ & $2-\mathrm{X}$ & $1,2-\mathrm{X}$ & $2,6-\mathrm{X}$ \\
\hline $\mathrm{X}=\mathrm{SH}$ & 5.29 & 5.31 & 5.14 & 5.30 \\
$\mathrm{X}=\mathrm{OH}$ & 6.71 & 6.76 & 6.64 & 6.69 \\
$\mathrm{X}=\mathrm{Br}$ & 5.70 & 5.80 & 5.23 & 5.81
\end{tabular}


We note that in terms of gap reduction relative to adamantane $\left(\Delta E_{\mathrm{vert}}=7.32 \mathrm{eV}\right.$, see above), the thiols are more efficient than the bromo compounds and alcohols are least efficient. In all cases the position of the substituent for mono-substitution (1 or 2) has only a minor effect and the introduction of a second substituent has a small additional effect on the gap for 2,6-, but a slight redshift for 1,2-substitution.

For all alcohols and bromo compounds listed in the table, we also computed vibronic absorption and emission spectra using the IMDHO (STA) model. As a result (not shown), one finds pronounced vibrational finestructures for 1- and 2-alcohols and 2,6-dialcohol, while that of 1,2-dialcohol and all bromo compounds appear to be unstructured. In case of the bromo-adamantanes, absorption and emission spectra are well separated, showing Stokes shifts between $1.3 \mathrm{eV}$ (2,6-dibromo) and $3.3 \mathrm{eV}$ (2-bromo). In fact, for 2-bromo-adamantane, the absorption maximum is at $5.8 \mathrm{eV}$ (and therefore almost equal to $\Delta E_{\text {vert }}$ ), while the emission spectrum has its maximum at around $2.5 \mathrm{eV}$ (around $500 \mathrm{~nm}$ ). Thus, emission is redshifted into the visible blue region at this level of theory. For comparison, the emission spectrum of adamantane has a maximum around $6 \mathrm{eV}$ (or $200 \mathrm{~nm}$ ), in the UV, according to a B3LYP/ TZVP/IMDHOFAD calculation. ${ }^{18}$ However, although our TD-B3LYP/ TZVP calculations of excited states did not converge, the $\mathrm{C}-\mathrm{Br}$ bond was seen to elongate considerably ( $0.22 \AA$ ) during the (incomplete) optimization, hence the $S_{1}$ state might actually be a dissociative state like the thiols, which is not expected to fluoresce. Experimental evidence would be very helpful.

\section{C-substituted diamondoids: urotropine and related compounds}

By replacing entire methylene and methine groups by heteroatoms we can realize another class of diamondoids, called "C-substituted" in this paper.

1. Urotropine. Hexamethylene tetramine $\left(\mathrm{CH}_{2}\right)_{6} \mathrm{~N}_{4}$, also known as urotropine, is perhaps the best known representative of this type, where all four methine $(\mathrm{CH})$ groups of adamantane are replaced by nitrogen $(\mathrm{N})$ atoms. Although urotropine retains the symmetry of adamantane ( $T_{\mathrm{d}}$ point group), the introduction of four nitrogen atoms alters its electronic properties ${ }^{14}$ compared to its purely hydrocarbon analogue.

The structure of the optimized (B3LYP/TZVP) ground state of urotropine is shown in Fig. 9. Several bond lengths and bond angles have also been mentioned there.

The $\mathrm{C}-\mathrm{H}$ and $\mathrm{C}-\mathrm{N}$ bond lengths are effectively unaltered in the optimized $\mathrm{S}_{1}$ state. The $\mathrm{H}-\mathrm{C}-\mathrm{H}$ bond angles increase by about $3^{\circ}$ and some $\mathrm{C}-\mathrm{N}-\mathrm{C}$ angles increase by about $4^{\circ}$, while some others decrease by $2^{\circ}$. The $\mathrm{N}-\mathrm{C}-\mathrm{N}$ angles decrease by about $3^{\circ}$, but one particular $\mathrm{N}-\mathrm{C}-\mathrm{N}$ bond angle decreases to $100^{\circ}$, i.e., by about $12^{\circ}$. As seen later, this will influence the absorption and rR spectra of urotropine.

Table 6 shows selected (mostly bright) vertical excitation energies out of the first ten singlet excited states of urotropine, from the $\mathrm{S}_{0}$ state, compared to analogous values for adamantane. ${ }^{18}$

The first allowed transition is to degenerate states $S_{1}, S_{2}$ and $\mathrm{S}_{3}$ (which are only nearly degenerate in numerical practice without symmetry restrictions). These transitions are dominated

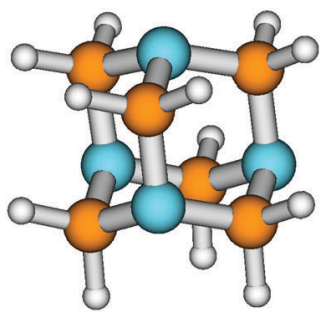

Fig. 9 The B3LYP/TZVP optimized ground state geometry of urotropine. The blue balls represent the $\mathrm{N}$ atoms, the brown balls the $\mathrm{C}$ atoms and the white balls represent the $\mathrm{H}$ atoms. The $\mathrm{C}-\mathrm{N}$ and $\mathrm{C}-\mathrm{H}$ bond lengths are 1.47 $\AA$ and $1.09 \AA$. The $\mathrm{C}-\mathrm{N}-\mathrm{C}$ and $\mathrm{H}-\mathrm{C}-\mathrm{H}$ bond angles are around $108^{\circ}$, while the $\mathrm{N}-\mathrm{C}-\mathrm{N}$ bond angles are $112.4^{\circ}$. Bond lengths and angles involving similar atoms are equal.

Table 6 In the upper half, vertical transition energies and oscillator strengths for selected states (labeled as $\alpha$ ) of urotropine and adamantane are shown. Energies are given up to four digits energies to demonstrate a slight degeneracy-loss in numerical calculations without symmetry restriction. The $\left(S_{1}\right)$ state which has been used for vibronic calculations is shown in bold. In the lower part of the table the $\Delta E_{0-0}, \Delta E_{\mathrm{HL}}$ and $\Delta E_{\mathrm{vibro}}$ energies are given for the $\mathrm{S}_{0} \rightarrow \mathrm{S}_{1}$ transition. Finally, $\Delta E_{\mathrm{opt}}$ (exp.) and $\Delta E_{\text {vibro }}$ (exp.) are the experimental optical gaps and vibronic maximum of the first absorption band, according to ref. 14. The experimental vibronic maximum of the first absorption band of adamantane has been obtained from ref. 10. All calculations were done on the B3LYP/TZVP/IMDHOFAD level of theory; all energies in eV

\begin{tabular}{|c|c|c|c|c|}
\hline \multirow[b]{2}{*}{ State $\alpha$} & \multicolumn{2}{|c|}{ Urotropine } & \multicolumn{2}{|c|}{ Adamantane } \\
\hline & $\omega_{\alpha}(\mathrm{eV})$ & $f_{\alpha}$ & $\omega_{\alpha}(\mathrm{eV})$ & $f_{\alpha}$ \\
\hline 1 & 6.0297 & 0.0024 & 7.3223 & 0.0064 \\
\hline 2 & 6.0300 & 0.0024 & 7.3227 & 0.0063 \\
\hline 3 & 6.0305 & 0.0024 & 7.3232 & 0.0064 \\
\hline$\dot{9}$ & 6.8818 & 0.0438 & 8.4546 & 0.0000 \\
\hline 10 & 6.8820 & 0.0438 & 8.4703 & 0.1040 \\
\hline$\Delta E_{0-0}$ & 5.42 & & 6.54 & \\
\hline$\Delta E_{\mathrm{HL}}$ & 6.92 & & 8.15 & \\
\hline$\Delta E_{\text {vibro }}$ & 5.80 & & 6.84 & \\
\hline$\Delta E_{\text {opt }}($ exp.) & 5.42 & & 6.49 & \\
\hline$\Delta E_{\text {vibro }}(\exp )$. & $\sim 6.3$ & & 6.8 & \\
\hline
\end{tabular}

by HOMO $\rightarrow$ LUMO excitations, the former being of $t_{2}$ symmetry in the $T_{\mathrm{d}}$ point group, the latter of $a_{1}$ symmetry. The next three transitions $\left(S_{4}\right.$ to $S_{6}$, not shown) are also three-fold degenerate but forbidden. At slightly higher energies $(\sim 6.9 \mathrm{eV})$, further bright states follow. The behavior is qualitatively analogous to adamantane. Quantitatively, the lowest-energy absorption of urotropine is redshifted relative to adamantane, and less intense.

For both compounds, a comparison to the experimental optical gap, i.e., the onset of absorption is possible. According to Table 6, the experimental optical gaps $\Delta E_{\text {opt }}$ (exp.) are close to the $\Delta E_{0-0}$ values calculated at the present level of theory. (For adamantane, this has been discussed already above.) For urotropine, a relevant comparison of the $\Delta E_{0-0}$ value can be made with the previously calculated absorption onset value of $5.80 \mathrm{eV}$ by Demján and co-workers. ${ }^{38} \Delta E_{0-0}$ predicts a redshift in absorption of about $1.1 \mathrm{eV}$, both in experiment ${ }^{14}$ and theory, if one considers indeed $\Delta E_{0-0}$ as a good measure for the optical gap. 
The agreement between theory and experiment is still good but less striking when fully vibrationally resolved absorption and emission spectra are considered instead. In Fig. 10 we show $S_{0} \leftrightarrow S_{1}$ vibronic absorption and emission spectra of urotropine, obtained on the B3LYP/TZVP/IMDHOFAD level of theory. Using the same Lorentzian broadening $\Gamma=200 \mathrm{~cm}^{-1}$ as in ref. 18, we find only a weak vibronic finestructure for absorption and emission of urotropine. This is contrast to pronounced finestructures found in theoretical spectra of adamantane in ref. 18 (Fig. 3 in that reference). Missing vibrational finestructure in the absorption spectrum of urotropine, but a clear finestructure for adamantane is also in full agreement with experiment. ${ }^{10,14}$ The same holds true for experimental emission spectra. ${ }^{10,14}$ A slight discrepancy between theory and experiment is found for the widths and maxima of the lowest-energy absorption and emission peaks of urotropine. For absorption, the lowest-energy band peaks at around $6.3 \mathrm{eV}$ according to ref. 14, with a FWHM value of about one eV. Our absorption band is slightly narrower according to Fig. 10 (FWHM $\sim 0.6 \mathrm{eV}$ ), with a maximum at about
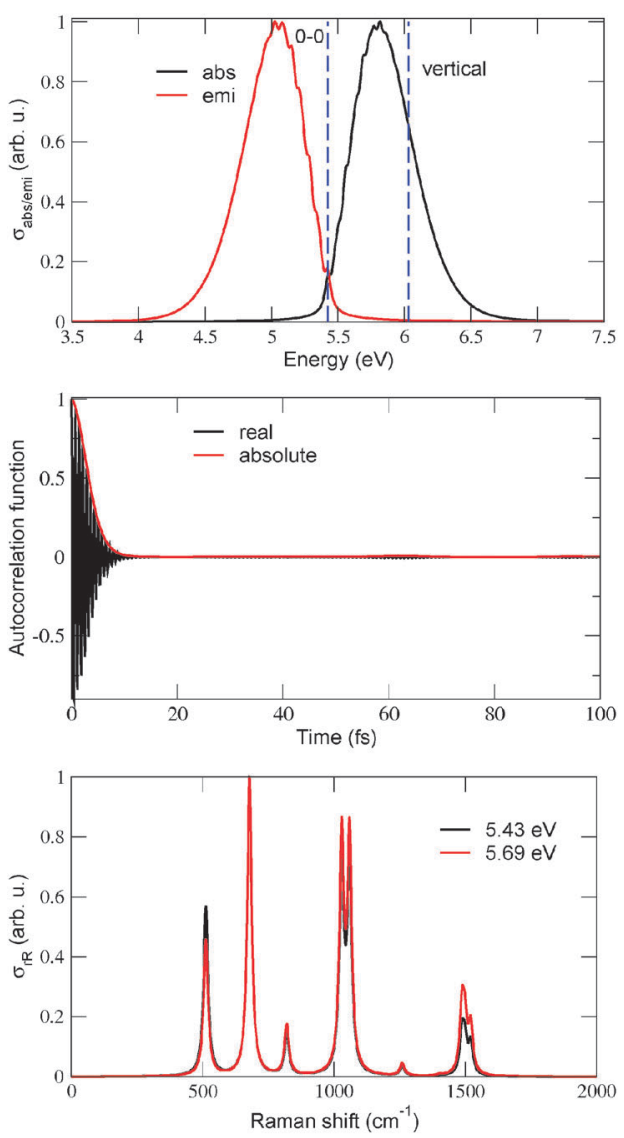

Fig. 10 The vibrationally resolved absorption and emission spectra (upper panel) for urotropine. Vertical dashed lines stand for $0-0$ and vertical (for absorption) transitions, respectively. The middle panel shows the corresponding autocorrelation function for absorption. For both upper panels damping factors $\Gamma=200 \mathrm{~cm}^{-1}$ have been used. Resonance Raman spectra at selected excitation energies $\omega_{\mathrm{L}}$ are shown in the lower panel. In this case, a broadening factor $\tilde{\Gamma}=200 \mathrm{~cm}^{-1}$ has been used and the resulting stick spectrum was broadened by normalized Lorentzians of FWHM of $10 \mathrm{~cm}^{-1}$. All calculations were at the (TD-)B3LYP/TZVP/IMDHOFAD level of theory.
$5.8 \mathrm{eV}$ (Table 6). The broader experimental spectrum may result from the presence of higher states around $6.88 \mathrm{eV}$ (Table 6) which have not been considered in our vibronic calculations. Similarly, our theoretical emission spectrum is redshifted by a few tenths of an $\mathrm{eV}$ and also narrower than the experimental one. ${ }^{14}$

The lack of a vibrational finestructure results from the usual fact that some normal modes (e.g. modes 6 and 10, discussed later) undergo quite high displacements during the transition. Consequently, the $0-0$ peak is also absent in the theoretical spectrum. The lack of vibrational finestructure in absorption is reflected in the lack of periodic recurrences in the absorption autocorrelation function (Fig. 10, middle panel), in contrast to adamantane, where clear recurrences are seen (Fig. 3(d) of ref. 18). In passing we note that using a lower broadening factor $\left(50 \mathrm{~cm}^{-1}\right)$ for calculating the absorption and emission spectra leads to the appearance of an extended finestructure in either case, from which little practical information about the possible contributing modes can be obtained, however. In this context, it might be relevant to mention that the choice of the broadening parameter depends essentially on the dimensionless origin shifts of the modes, which in turn, depends on the extent of geometrical distortion between the ground and excited states. Usually, for the pristine diamondoids, a FWHM of $200 \mathrm{~cm}^{-1}$ has been found to give good (compared to experiments) spectral resolution, e.g., in ref. 18 . However, since most of the functionalized diamondoids involve considerably high degree of geometric distortions in the excited states, many normal modes have been found to exhibit high values of dimensionless origin-shifts, hence we had to use a lower broadening factor of FWHM $=50-100 \mathrm{~cm}^{-1}$ in most of these cases.

From the previous results, it was seen that resonance Raman spectra can provide an indication of the vibrational modes excited during electronic excitation in molecules. Therefore, the resonance Raman spectrum of urotropine was calculated and used to get a better insight into the normal modes involved in the excitation process. Fig. 10, lower panel shows the resonance Raman spectra at two different excitation energies corresponding to selected values in the resonance region. The spectra are featured by intense peaks around 510, 675, 1024, 1050,1485 and $1500 \mathrm{~cm}^{-1}$. These peaks originate from modes $6,10,23,25$ and three nearly spaced modes 43,46 and 48 . The first four modes correspond to the vibrations of the sixmembered rings and the $\mathrm{N}-\mathrm{C}-\mathrm{N}$ units and the last three are the various bending vibrations of the different methylene units. The intensities of the peaks corresponding to the methylene vibrations are lower than those which originate from ring torsional and $\mathrm{N}-\mathrm{C}-\mathrm{N}$ vibrational modes. The excitation of the $\mathrm{N}-\mathrm{C}-\mathrm{N}$ vibrations and some of these modes showing high values of shifts can be attributed to the change in the $\mathrm{N}-\mathrm{C}-\mathrm{N}$ bond angles during electronic excitation. This is again due to the HOMO now being centered on the nitrogen atoms. Hence, alteration of electronic structure of diamondoids can result in the alteration of their vibrational characteristics.

2. Related compounds. We also studied adamantane derivatives in which not all four, but only one, two, or three methine units were replaced by $\mathrm{N}$. This gives rise to monoaza, diaza and 
Table 7 The HOMO-LUMO gap $\Delta E_{\mathrm{HL}}, 0-0$ transition energy $\Delta E_{0-0}$, vertical transition energy $\Delta E_{\text {vert }}$ and main maximum of the vibronic absorption spectrum $\Delta E_{\text {vibro }}$ for various oxa and aza compounds (see text), calculated on the B3LYP/TZVP/IMDHO (STA) level of theory. The corresponding values for adamantane are also shown, computed on the B3LYP/TZVP/IMDHOFAD level of theory. All reported energies are in eV

\begin{tabular}{lllll}
\hline Molecule & $\Delta E_{\mathrm{HL}}$ & $\Delta E_{0-0}$ & $\Delta E_{\mathrm{vert}}$ & $\Delta E_{\mathrm{vibro}}$ \\
\hline Adamantane & 8.15 & 6.54 & 7.32 & 6.84 \\
& & & & \\
Monooxa & 7.13 & 6.17 & 6.29 & 6.17 \\
2,4-Dioxa & 7.44 & 6.36 & 6.60 & 6.36 \\
2,6-Dioxa & 7.52 & 6.48 & 6.61 & 6.48 \\
2,4,6-Trioxa & 7.83 & 6.85 & 6.92 & 6.85 \\
2,4,10-Trioxa & 7.66 & 6.62 & 6.81 & 6.62 \\
Tetraoxa & 9.28 & 7.13 & 7.39 & 7.27 \\
& & & & \\
Monoaza & 6.11 & 4.97 & 5.31 & 5.21 \\
Diaza & 6.05 & 4.95 & 5.23 & 5.16 \\
Triaza & 6.45 & 5.28 & 5.60 & 5.49 \\
Urotropine & 6.92 & 5.62 & 6.03 & 5.97 \\
& & & &
\end{tabular}

triaza adamantanes. Further, one may also replace methylene $\left(\mathrm{CH}_{2}\right)$ units of adamantane, by atoms like oxygen $(\mathrm{O})$. We considered adamantanes with one (monooxa), two (2,4-dioxa, 2,6-dioxa), three (2,4,6-trioxa, 2,4,10-trioxa) and four (tetraoxa adamantane) oxygens, respectively. In Table 7 we list $\Delta E_{\mathrm{HL}}, \Delta E_{0-0}, \Delta E_{\text {vert }}$ and $\Delta E_{\text {vibro }}$ values for absorption, for all of these compounds. The B3LYP/TZVP method was used again and in case of vibronic spectra, for simplicity the IMDHO (STA) method was adopted.

From the table we note that for aza compounds, optical gaps do not linearly decrease with the degree of $\mathrm{N}$ substitution. Rather, the diaza compound shows the lowest-energy absorption, followed by monoaza, triaza and urotropine. Also for oxa compounds the perhaps expected trend that more oxygens simply lead to lower absorption energies does not hold. Rather, the lowest optical gap is obtained for monooxa, followed by the di- and tri-oxas and the tetraoxa adamantane in this case. Tetraoxa adamantane is in fact an example, where the optical absorption is predicted to be blueshifted with respect to the parent compound, adamantane. We also note that our calculated $\Delta E_{0-0}$ value $(6.17 \mathrm{eV})$ of monooxa adamantane is in good agreement with the measured optical gap $(6.18 \mathrm{eV})$ given in ref. 37.

\section{Summary and outlook}

In this work, a time-dependent approach to vibronically resolved spectroscopy based on autocorrelation and cross-correlation functions was applied, along with time-dependent (linearresponse) density functional theory to understand certain optical properties of electronically blended, functionalized and "doped" (or "C-substituted") diamondoids. In particular, absorption, emission and resonance Raman spectra were computed. The Condon and harmonic approximations were used for this. The IMDHO (STA $)^{29}$ and IMDHOFAD models ${ }^{18,25}$ have been used for the calculation of time-dependent correlation functions. In cases when large-amplitude motions and large displacements in excited states take place, the IMDHOFAD may be difficult to realize. Fortunately in these cases a "vertical" approach like
IMDHO (STA) could also be more realistic. If one or the other approach is more appropriate, is generally still an open question.

The absorption and emission spectra of the modified diamondoids are (almost all) redshifted with respect to the pristine diamondoids. The magnitude and trends of the redshift depends on the nature of the frontier orbitals which are involved in the electronic transition. This, again, depends on the nature, number and position of functional groups or substituents attached to the diamondoids. With adequate number of thione groups, for example, the absorption can be shifted to the visible region. Geometrical distortions during the electronic excitation also affect the resolution of the spectra. Important information about the vibrational normal modes playing dominant roles in the electronic excitation were extracted from the resonance Raman spectra, particularly in situations where well resolved absorption spectra could not be obtained due to significant geometrical distortions. Where comparison to experiment and previous theoretical results was possible, the present theory gave reasonable agreement. We made, however, also a large number of predictions which await experimental proof. It must be said, though, that simple explanations for observed trends are not always available. In these cases simulations are needed and therefore sufficiently accurate and at the same time sufficiently economic theoretical methods, to allow for systematic investigations of a large class of candidate materials. We believe that the present methodology is a suitable starting point.

Of course, the present methods could and should be improved. The application of more detailed basis sets and/or more accurate electronic structure methods is a worthwhile direction. Effects of anharmonicity and finite temperature might also be studied. Further, the inclusion of non-radiative transitions such as intersystem crossing by spin-orbit coupling, or internal conversion due to non-Born-Oppenheimer couplings, will be interesting to include. In this context it should be noted that the time-dependent correlation function approach may offer advantages over traditional, timeindependent (Golden Rule type) approaches, too. ${ }^{41,42}$

\section{Acknowledgements}

We sincerely thank Dr Dominik Kröner, Tao Xiong (Potsdam) as well Thomas Möller and Janina Maultzsch (TU Berlin) for fruitful discussions. Financial support by the "DFG-Forschergruppe 1282" (project Sa 547/11-1) is gratefully acknowledged.

\section{References}

1 J. E. Dahl, S. G. Liu and R. M. K. Carlson, Science, 2003, 299, 96.

2 P. R. Schreiner, L. V. Chernish, P. A. Gunchenko, E. Y. Tikhonchukh, H. Hausmann, M. Serafin, S. Schlecht, J. E. P. Dahl, R. M. K. Carlson and A. A. Fokin, Nature, 2011, 477, 308.

3 H. Schwertfeger and P. R. Schreiner, Chem. Unserer Zeit, 2010, 44, 248. 
4 H. Schwertfeger, A. A. Fokin and P. R. Schreiner, Angew. Chem., Int. Ed., 2008, 47, 1022.

5 W. L. Yang, J. D. Fabbri, T. M. Willey, J. R. I. Lee, J. E. Dahl, R. M. K. Carlson, P. R. Schreiner, A. A. Fokin, B. A. Tkachenko, N. A. Fokina, W. Meevasana, N. Mannella, K. Tanaka, X. J. Zhou, T. van Burren, M. A. Kelly, Z. Hussain, N. A. Melosh and Z. X. Shen, Science, 2007, 316, 1460.

6 S. Roth, D. Leunberger, J. Osterwalder, J. E. Dahl, R. M. K. Carlson, B. A. Tkachenko, A. A. Fokin, P. R. Schreiner and M. Hengsberger, Chem. Phys. Lett., 2010, 495, 102.

7 W. A. Clay, Z. Liu, W. Yang, J. D. Fabbri, J. E. Dahl, R. M. K. Carlson, Y. Sun, P. R. Schreiner, A. A. Fokin, B. A. Tkachenko, N. A. Fokina, P. A. Pianetta, N. Melosh and Z.-X. Shen, Nano Lett., 2009, 9, 57.

8 L. Landt, K. Klünder, J. E. Dahl, R. M. K. Carlson, Th. Möller and Ch. Bostedt, Phys. Rev. Lett., 2009, 103, 047402.

9 M. Steglich, F. Husiken, J. E. Dahl, R. M. K. Carlson and Th. Henning, Astrophys. J., 2011, 729, 1.

10 R. Richter, D. Wolter, T. Zimmermann, L. Landt, A. Knecht, C. Heidrich, A. Merli, O. Dopfer, P. Reiß, A. Ehresmann, J. Petersen, J. E. Dahl, R. M. K. Carlson, C. Bostedt, T. Möller, R. Mitrić and T. Rander, Phys. Chem. Chem. Phys., 2014, 16, 3070.

11 R. Meinke, R. Richter, A. Merli, A. A. Fokin, T. V. Koso, V. N. Rodionov, P. R. Schreiner, C. Thomsen and J. Maultzsch, J. Chem. Phys., 2014, 140, 034309.

12 A. A. Fokin and P. R. Schreiner, Mol. Phys., 2009, 107, 823.

13 A. A. Fokin, E. D. Butova, L. V. Chernish, N. A. Fokina, J. E. P. Dahl, R. M. K. Carlson and P. R. Schreiner, Org. Lett., 2007, 9, 2541.

14 L. Landt, W. Kieleich, D. Wolter, M. Staiger, A. Ehresmann, Th. Möller and Ch. Bostedt, Phys. Rev. B: Condens. Matter Mater. Phys., 2009, 80, 205323.

15 L. Landt, M. Staiger, D. Wolter, K. Klünder, P. Zimmermann, T. M. Willey, T. van Bluren, D. Brehmer, P. R. Schreiner, B. A. Tkachenko, A. A. Fokin, Th. Möller and Ch. Bostedt, J. Chem. Phys., 2010, 132, 024710.

16 L. Landt, C. Bostedt, D. Wolter, Th. Möller, J. E. P. Dahl, R. M. K. Carlson, B. A. Tkachenko, A. A. Fokin, P. R. Schreiner, A. Kulesza, R. Mitrić and V. Bonačić-Koutecký, J. Chem. Phys., 2010, 132, 144305.

17 T. Rander, M. Steiger, R. Richter, T. Zimmermann, L. Landt, D. Wolter, J. E. Dahl, R. M. K. Carlson, B. A. Tkachenko, N. A. Fokina, P. R. Shreiner, T. Möller and C. Bostedt, J. Chem. Phys., 2013, 138, 024310.

18 S. Banerjee and P. Saalfrank, Phys. Chem. Chem. Phys., 2014, 16, 144.

19 T. Zimmermann, R. Richter, A. Knecht, A. A. Fokin, T. V. Koso, L. V. Chernish, P. A. Gunchenko, P. R. Schreiner, T. Möller and T. Rander, J. Chem. Phys., 2013, 139, 084310.

20 B. A. Tkachenko, N. A. Fokina, L. V. Chernish, J. E. P. Dahl, S. Liu, R. M. K. Carlson, A. A. Fokin and P. R. Schreiner, Org. Lett., 2006, 8, 1767.

21 A. A. Fokin, T. S. Zhuk, A. E. Pashenko, P. O. Dral, P. A. Gunchenko, J. E. P. Dahl, R. M. K. Carlson,
T. V. Koso, M. Serafin and P. R. Schreiner, Org. Lett., 2009, 11, 3068.

22 M. Vörös, T. Demján, T. Szilvási and A. Gali, Phys. Rev. Lett., 2012, 108, 267401.

23 S. Y. Lee and E. J. Heller, J. Chem. Phys., 1979, 71, 4777.

24 D. J. Tannor and E. J. Heller, J. Chem. Phys., 1982, 77, 202.

25 S. Banerjee, D. Kröner and P. Saalfrank, J. Chem. Phys., 2012, 137, 22A534.

26 H. Ma, J. Liu and W. Liang, J. Chem. Theory Comput., 2012, 8, 4474.

27 A. Baiardi, J. Bloino and V. Barone, J. Chem. Phys., 2014, 141, 114108.

28 J. P. Götze, B. Karasulu and W. Thiel, J. Chem. Phys., 2013, 139, 234108.

29 T. Petrenko and F. Neese, J. Chem. Phys., 2007, 127, 164319. 30 A. D. Becke, J. Chem. Phys., 1993, 98, 5648.

31 C. Lee, W. Yang and R. G. Parr, Phys. Rev. B: Condens. Matter Mater. Phys., 1988, 37, 785.

32 A. Schäfer, C. Huber and R. Ahlrichs, J. Chem. Phys., 1994, 100, 5829.

33 M. J. Frisch, G. W. Trucks, H. B. Schlegel, G. E. Scuseria, M. A. Robb, J. R. Cheeseman, G. Scalmani, V. Barone, B. Mennucci, G. A. Petersson, H. Nakatsuji, M. Caricato, X. Li, H. P. Hratchian, A. F. Izmaylov, J. Bloino, G. Zheng, J. L. Sonnenberg, M. Hada, M. Ehara, K. Toyota, R. Fukuda, J. Hasegawa, M. Ishida, T. Nakajima, Y. Honda, O. Kitao, H. Nakai, T. Vreven, J. A. Montgomery, Jr., J. E. Peralta, F. Ogliaro, M. Bearpark, J. J. Heyd, E. Brothers, K. N. Kudin, V. N. Staroverov, R. Kobayashi, J. Normand, K. Raghavachari, A. Rendell, J. C. Burant, S. S. Iyengar, J. Tomasi, M. Cossi, N. Rega, J. M. Millam, M. Klene, J. E. Knox, J. B. Cross, V. Bakken, C. Adamo, J. Jaramillo, R. Gomperts, R. E. Stratmann, O. Yazyev, A. J. Austin, R. Cammi, C. Pomelli, J. W. Ochterski, R. L. Martin, K. Morokuma, V. G. Zakrzewski, G. A. Voth, P. Salvador, J. J. Dannenberg, S. Dapprich, A. D. Daniels, Ö. Farkas, J. B. Foresman, J. V. Ortiz, J. Cioslowski and D. J. Fox, Gaussian 09 (Revision A.02), Gaussian, Inc, Wallingford, CT, 2009.

34 M. Frigo and S. G. Johnson, Proc. IEEE, 2005, 93(2), 216. 35 F. Neese, ORCA, an ab initio, density functional and semiempirical program package, University of Bonn, Bonn, Germany, 2007.

36 T. S. Zhuk, T. Koso, A. E. Pashenko, N. T. Hoc, V. N. Rodionov, M. Serafin, P. R. Schreiner and A. A. Fokin, J. Am. Chem. Soc., 2015, 137, 6577.

37 L. Landt, Electronic structure and optical properties of pristine and modified diamondoids, PhD thesis, Technical University of Berlin, 2010.

38 T. Demján, M. Vörös, M. Palummo and A. Gali, J. Chem. Phys., 2014, 141, 064308.

39 G. Strinati, Phys. Rev. Lett., 1982, 49, 1519.

40 K. J. Falk and R. P. Steer, Can. J. Chem., 1988, 66, 575.

41 M. Etinski, J. Tatchen and C. M. Marian, J. Chem. Phys., 2011, 134, 154105.

42 Y. Niu, Q. Peng, Ch. Deng, X. Gao and Z. Shuai, J. Phys. Chem. A, 2010, 114, 7817. 